U. S. Department of the Interior

U. S. Geological Survey

\title{
Petroleum Systems of the Po Basin Province of Northern Italy and the Northern Adriatic Sea: Porto Garibaldi (Biogenic), Meride/Riva di Solto (Thermal), and Marnoso Arenacea (Thermal)
}

\author{
On-Line Edition
}

by

Sandra J. Lindquist ${ }^{1}$

\section{Open-File Report 99-50-M}

This report is preliminary and has not been reviewed for conformity with the U.S. Geological Survey editorial standards or with the North American Stratigraphic Code. Any use of trade names is for descriptive purposes only and does not imply endorsement by the U.S. government.

1999

${ }^{1}$ Consulting Geologist, Contractor to U. S. Geological Survey, Denver, Colorado 


\title{
Petroleum Systems of the Po Basin Province of Northern Italy and the Northern Adriatic Sea: Porto Garibaldi (Biogenic), Meride / Riva di Solto (Thermal), and Marnoso Arenacea (Thermal) ${ }^{2}$
}

\author{
Sandra J. Lindquist, Geological Consultant, \\ Contractor to U.S. Geological Survey, Denver, CO
}

November, 1999

\section{FOREWORD}

This report was prepared as part of the World Energy Project of the U.S. Geological Survey. In the project, the world was divided into eight regions and 937 geologic provinces. The provinces have been ranked according to the discovered oil and gas volumes within each (Klett and others, 1997). Then, 76 "priority" provinces (exclusive of the U.S. and chosen for their high ranking) and 26 "boutique" provinces (exclusive of the U.S. and chosen for their anticipated petroleum richness or special regional economic importance) were selected for appraisal of oil and gas resources. The petroleum geology of these priority and boutique provinces is described in this series of reports.

Asssessment results will be available later. The Po Basin Province ranks 68th in the world, exclusive of the U.S, with respect to ultimate recoverable hydrocarbons.

The purpose of this effort is to aid in assessing the quantities of oil, gas, and natural gas liquids that have the potential to be added to reserves within the next 30 years. These volumes either reside in undiscovered fields whose sizes exceed the stated minimumfield-size cutoff value for the assessment unit (variable, but must be at least 1 million barrels of oil equivalent) or occur as reserve growth of fields already discovered.

The total petroleum system constitutes the basic geologic unit of the oil and gas assessment. The total petroleum system includes all genetically related petroleum occurring in shows and accumulations (discovered and undiscovered) that has been generated by a pod or by closely related pods of mature source rock and that exists within a limited mappable geologic space, together with the essential mappable geologic

\footnotetext{
${ }^{2}$ Porto Garibaldi Total Petroleum System (\#406001) (Miocene/Pliocene/Pleistocene biogenic gas); Po Basin Province (\#4060); Europe (Region 4); Northern Italy and Northern Adriatic Sea (note that for the quantitative resource assessment, this total petroleum system is combined with Marnoso Arenacea); Meride / Riva di Solto Total Petroleum System (\#406002) (Triassic thermal oil and gas); Po Basin Province (\#4060); Europe (Region 4); Northern Italy and Northern Adriatic Sea; Marnoso Arenacea Total Petroleum System (no number assigned) (Tertiary thermal gas and oil); Po Basin Province (\#4060); Europe (Region 4); Northern Italy and Northern Adriatic Sea. Note that for the quantitative resource assessment, this total petroleum system is included with 406001.
} 
elements (source, reservoir, seal, and overburden rocks) that control the fundamental processes of generation, expulsion, migration, entrapment, and preservation of petroleum. The total petroleum system concept is modified from Magoon and Dow, 1994. The minimum petroleum system is that part of a total petroleum system encompassing discovered shows and accumulations, together with the geologic space in which the various essential elements have been proved by these discoveries.

An assessment unit is a mappable part of a total petroleum system in which discovered and undiscovered fields constitute a single relatively homogenous population such that the chosen methodology of resource assessment - based on estimation of the number and sizes of undiscovered fields - is applicable. A total petroleum system might equate to a single assessment unit. If necessary, a total petroleum system may be subdivided into two or more assessment units such that each assessment unit is sufficiently homogeneous in terms of geology, exploration considerations, and risk to assess individually. Assessment units are considered established if they contain more than 13 fields, frontier if they contain 1-13 fields, and hypothetical if they contain no fields.

A graphical depiction of the elements of the total petroleum system is provided in the form of an events chart that shows the time of deposition of essential rock units; the time processes, such as trap formation, necessary for the accumulation of hydrocarbons; the critical moment in the total petroleum system; and the preservation time, if any.

A numeric code identifies each region, province, total petroleum system, and assessment unit; these codes are uniform throughout the project and will identify the same item in any of the publications. The code is as follows:

Region, single digit

Example

Province, three digits to the right of region code

$\underline{\mathbf{3}}$

Total Petroleum System, two digits to the right of province code $3162 \underline{\mathbf{0 5}}$

Assessment unit, two digits to the right of petroleum system code $316205 \underline{04}$ The codes for the regions and provinces are listed in Klett and others, 1997.

Oil and gas reserves quoted in this report are derived from the Petroleum Exploration and Production database (Petroconsultants, 1996) and other area reports from Petroconsultants, Inc., unless otherwise noted.

Figure(s) in this report that show boundaries of the total petroleum system(s), assessment units, and pods of active source rocks were compiled using geographic information system (GIS) software. Political boundaries and cartographic representations were taken, with permission, from Environmental Systems Research Institute's ArcWorld 1:3 million digital coverage (1992), have no political significance, and are displayed for general reference only. Oil and gas field centerpoints, shown on this(these) figure(s), are reproduced, with permission, from Petroconsultants, 1996. 


\section{$\underline{\text { ABSTRACT }}$}

The Porto Garibaldi total petroleum system dominates the Po Basin Province of onshore northern Italy and offshore Italy and Croatia in the northern Adriatic Sea. Porto Garibaldi contains Pliocene (primarily) and Pleistocene (secondarily) biogenic gas - approximately $16 \mathrm{TCF}$ (2.66 BBOE) ultimately recoverable - accumulated in co-eval siliciclastic reservoirs. This area was the northwestern edge of the Gondwanan (African) continental plate in pre-Hercynian time until the assembly of Pangea, a dominantly carbonate passive continental margin during the Mesozoic breakup of Pangea, and a Cenozoic collision zone with siliciclastic foredeep and foreland regions surrounded by thrust belts. At least two other petroleum systems, with Triassic (Meride / Riva di Solto) and Miocene (Marnoso Arenacea) source rocks, contribute oil and thermal gas reserves (nearly 1 $\mathrm{BBOE}$ ) to the province. The major time of hydrocarbon expulsion of the thermal systems was Late Neogene during the Alpine and Apennine orogenies. Local Mesozoic oil expulsion from Triassic rocks also occurred, but those oils either were not trapped or were leaked from faulty traps through time.

\section{INTRODUCTION}

The majority of hydrocarbons in the Po Basin Province (Figure 1) are Pliocene and Pleistocene (with minor Miocene sourced and reservoired) biogenic/diagenetic gas. Additional hydrocarbon contributions are from thermogenic, Mesozoic- and Miocenesourced and reservoired gas and oil. Two of the three total petroleum systems are assigned numbers in order of volumetric importance (406001=biogenic Plio/Pleistocene (Porto Garibaldi) and 406002=thermal Triassic (Meride / Riva di Solto)). The third small total petroleum system (thermal Tertiary (Marnoso Arenacea) is combined with 406001 for the purposes of quantitative resource assessment. That composite total petroleum system retains the number 406001 and the name Porto Garibaldi in the U.S. Geological Survey data base.

Within the body of this report, discussions of geology or petroleum system components occasionally occur in an order reflecting geologic age instead of volumetric significance. References listed in this report include a limited selection of those most recent and most pertinent to this document. Not all are specifically cited in the text. The stratigraphic equivalents chart (Figure 2) is composited from many references to approximately equate the range of stratigraphic nomenclature in use. It is not intended to be precise with respect to absolute geologic age. All stated reserves are derived from Petroconsultants (1996), unless referenced otherwise, and are "ultimate recoverable" volumes rather than "in place" numbers. 
Figure 2. Post-Hercynian stratigraphic equivalents of the Po Basin Province.

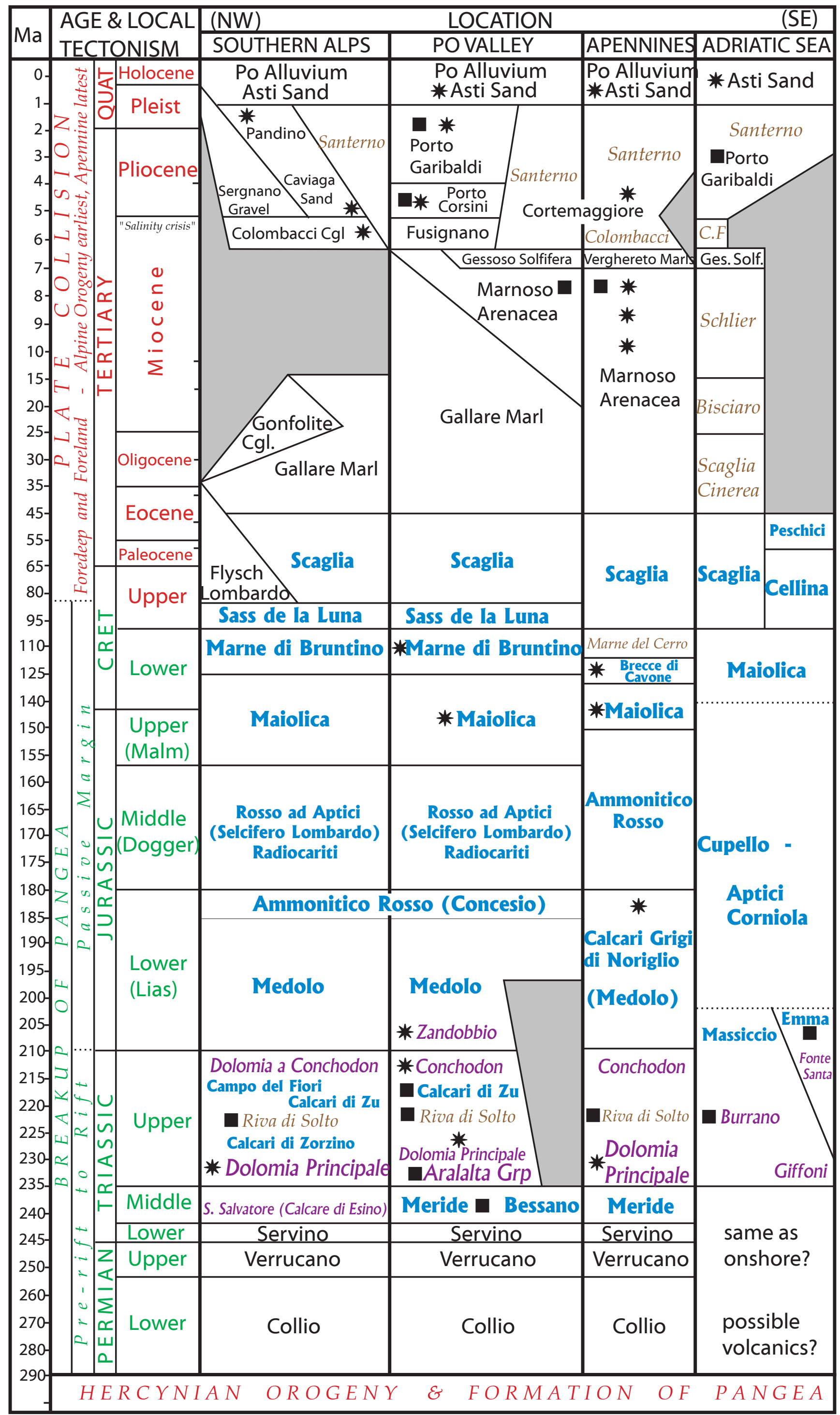

EXPLANATION * Producing reservoir Mostly Limestone Mostly Shale

Potential source rock Mostly Dolomite Miocene Gessoso Solfifera has volcanics 


\section{PROVINCE GEOLOGY}

\section{Province Boundary and Geographic Setting}

The 115,000-sq-km Po Basin Province of southern Europe is located between $43^{\circ}-46^{\circ}$ north latitude and $7^{\circ}-15^{\circ}$ east longitude (Figure 1). It includes northern Italy's Lombardy, Po River, and Veneto plains, as well as the northern Adriatic Sea with an adjacent portion of onshore Croatia (formerly Yugoslavia) called the Istrian Peninsula. Approximately two-thirds of the area is onshore (98\% Italy, 2\% Croatia) and one-third offshore (65\% Italy, 35\% Croatia). A very small portion of the Istrian offshore might technically belong to Slovenia. The province is surrounded by thrusted terrain of the southern Alps Mountains to the west and north, the northern Apennine Mountains to the south, and the Dinaride Mountains to the east. The province contains limited south-verging structural deformation on the Adriatic and Pedealpine homoclines; significant north-verging deformation in the Monferrato, Emilia, Ferrara, Romagna and Adriatic fold systems; and limited west-verging deformation from the Dinaride thrust system. The small country of San Marino is included in the southern part of the province.

\section{Political Entities}

Italy has favorable regulations for industry and has just recently opened its prospective lands to leasing by public and private foreign companies. Croatia and the other formerYugoslavian republics are involved in continuing disputes amongst themselves. The most recent Croatian wells were completed in 1990 (Petroconsultants, 1996).

\section{Geologic Setting}

Prior to the Late Paleozoic Hercynian orogeny and creation of the Pangea megacontinent, the Po Basin Province was on the northwestern edge of the Gondwana (Africa) subcontinent (Pieri and Flores, 1996; Dercourt and others, 1993; Anelli and others, 1986; Mattavelli and others, 1983). Deformation associated with Hercynian suturing has resulted in pre-Hercynian, Paleozoic rocks having poor reservoir potential, and they are referred to as "basement."

A post-Hercynian tensional regime affected the central Mediterranean area with the opening of the NeoTethyan and Mesogean oceanic basins. The Po Basin Province was part of the Adriatic ${ }^{3}$ microplate system developed between Africa and Europe. Early Mesozoic rocks record this breakup of Pangea - as well as an overall Mesozoic rising sea level - with pre-rift, Permo-Triassic continental siliciclastic rocks grading upward into Triassic rift sandstones, siltstones, shales, evaporites and carbonates (Anelli and others, 1996). North-south trending, early Mesozoic shallow-water carbonate platforms were laterally separated by extensional troughs, including the Olenus-Pindos, Sub-Pelagonian, Lagonegro, S. Alpine, Austroalpine and Piedmont rifts, in which organic-carbon rich sediments accumulated. The most anoxic basins with the richest organic matter were

${ }^{3}$ associated plate names are Adria, Apulia, Dinarid 
established in the Late Triassic Period. Platform and trough locations were relatively consistent throughout the Mesozoic era.

Jurassic and Cretaceous strata contain evidence for a continuous transition to a passive continental margin with shallow-water carbonates grading upward into generally deeperwater limestones and cherts. Regional stress regimes changed as the central and northern Atlantic Ocean began to open. Late Cretaceous to Cenozoic subduction of Tethys oceanic crust and continental collision between Africa and Europe partly deformed, rotated, overrode and consumed the Adriatic microplate, but a significant portion of that plate was preserved as a minimally deformed foreland region under the central portion of the Adriatic Sea and under the Po River basin. The area evolved into a deep-water basin characterized by siliciclastic turbidite and hemipelagite deposition that was impacted by continued compressional events and Tertiary sea level fluctuations.

The earliest evidence for Alpine compression is in the northern Alps Mountains, where northward thrusting is recorded in Upper Cretaceous Lombardo flysch (Mattavelli and others, 1983). By Oligocene time, most of the intervening part of the Tethys Ocean had been subducted, and the Adria continental microplate was being compressed from several directions. Change in relative plate motion was causing southward thrusting in the southern Alps and westward thrusting in the Dinarides, the latter attaining their present location at the eastern coast of the Adriatic Sea (Figures 3a, 3b, 3c, 3d). Also beginning in the Oligocene Epoch in westerly distal locations was an eastward compressional stacking of carbonate platforms and their associated deeper basins to form the earliest Apennines (Haan and Arnott, 1991). This deformation would reach the west side of this province 10-15 m.y. later by Middle Miocene time.

A Late Miocene (Messinian) drop in sea level, combined with the effects of continued Euro-African collision, resulted in periodic isolation of the Mediterranean basin from open marine circulation. Local unconformities developed, and widespread evaporites were deposited. The Apennine orogeny peaked in Pliocene time, with the deformed zone reaching its northeasternmost expression across much of Italy, although some deformation continued into the Pleistocene Epoch. Substantial foredeep subsidence resulted in the deposition of at least $6 \mathrm{~km}$ of Pliocene sediment (Haan and Arnott, 1991), the subsequent filling of the foredeep, and the establishment of the Po River drainage system. Transpressional deformation continues, but certain foreland areas - especially along the Croatian Adriatic Sea and Istrian Peninsula - still remain relatively undeformed.

\section{Exploration History}

Hydrocarbon shows in the Italian Apennines have been known since the 1700s, and some shallow wells there were drilled as early as 1860. The first exploratory wells in the Po Basin Province of Italy were drilled in 1890 and 1907, followed by four more in the 1920s. Generally, several to ten Italian exploratory wells were drilled annually during the 1930s and 1940s, and both exploration and development operations intensified in the 
Figure 3a. Western onshore structural cross section, Po Basin Province, Northern Italy (after Anelli and others, 1996).

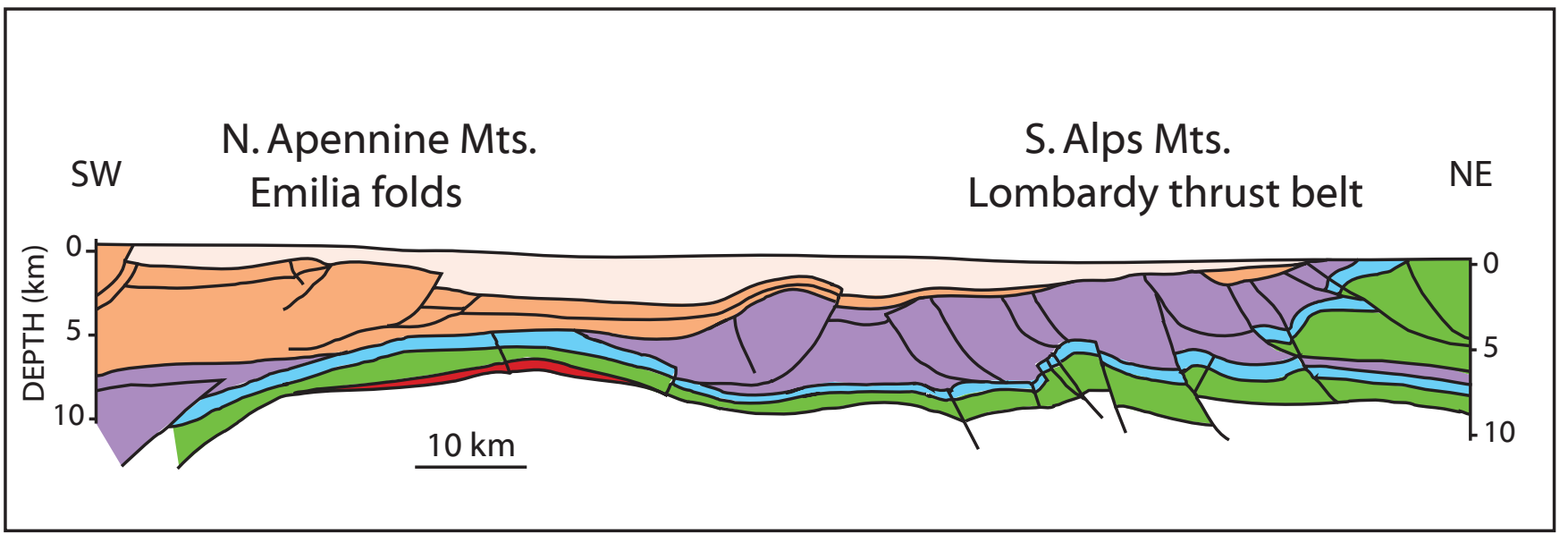

\section{LEGEND (Figures 3a \& 3b)}

Middle Pliocene - Quaternary

Latest Miocene - Eary Pliocene

Paleogene - Late Miocene

Triassic - Cretaceous deep marine

Triassic - Cretaceous shallow marine

Permian - Early Triassic siliciclastics

Volcanics

Cross section locations on Figure 1

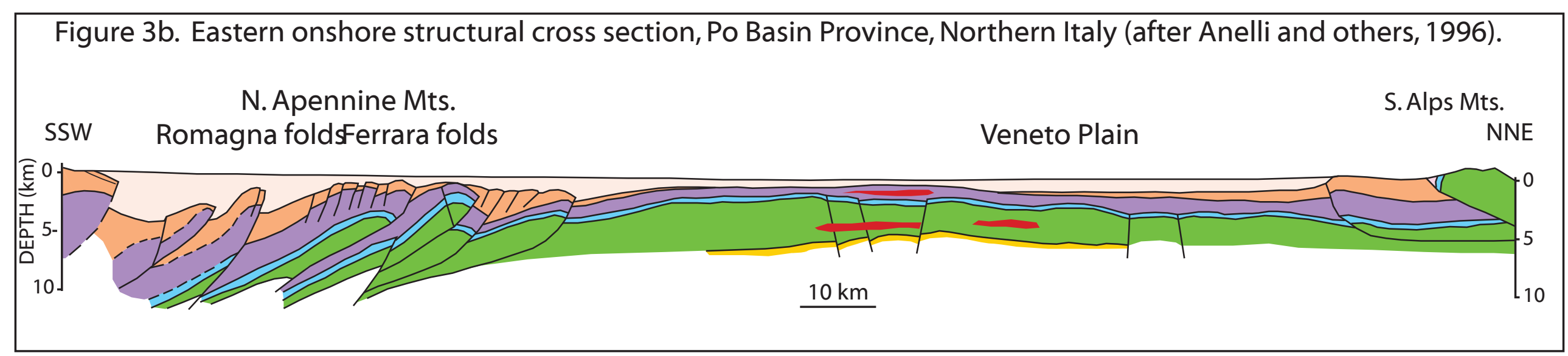


Figure 3c. Northern offshore structural cross section, Po Basin Province, northern Adriatic Sea (after Mattavelli and others, 1991).

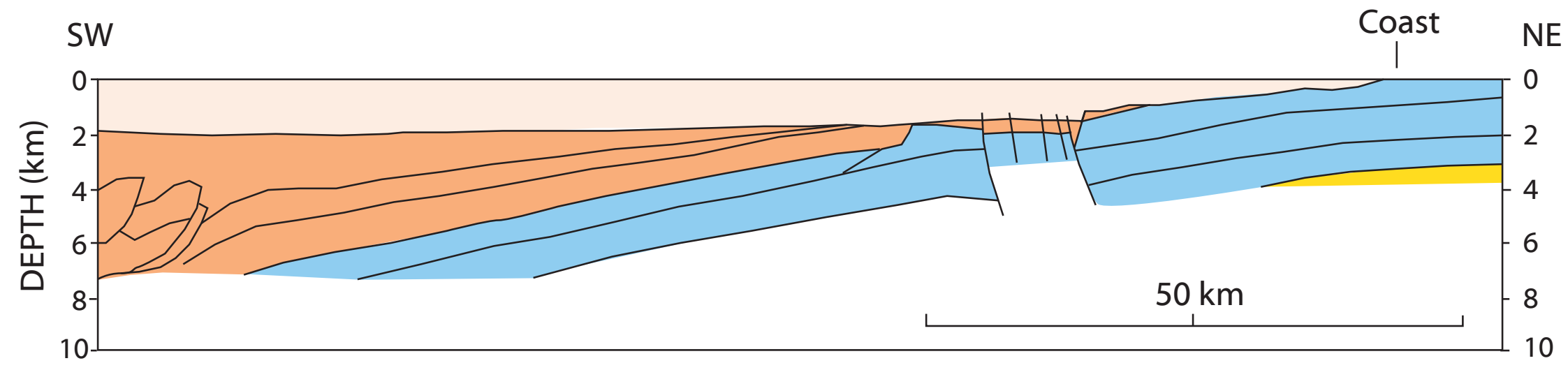

$\begin{array}{llll}\text { LEGEND } & \square \text { Cretaceous - Triassic carbonates } & \\ \text { (Figures } & \square \text { Pliocene } & \square \text { Permian siliciclastics } & \text { Cross section } \\ \text { 3c \& 3d) } & \square \text { Pre-Pliocene Tertiary } & \square \text { Volcanics } & \text { locations on } \\ & & \end{array}$

Figure 3d. Southern offshore structural cross section, Po Basin Province, north-central Adriatic Sea (after Mattavelli and others, 1991).

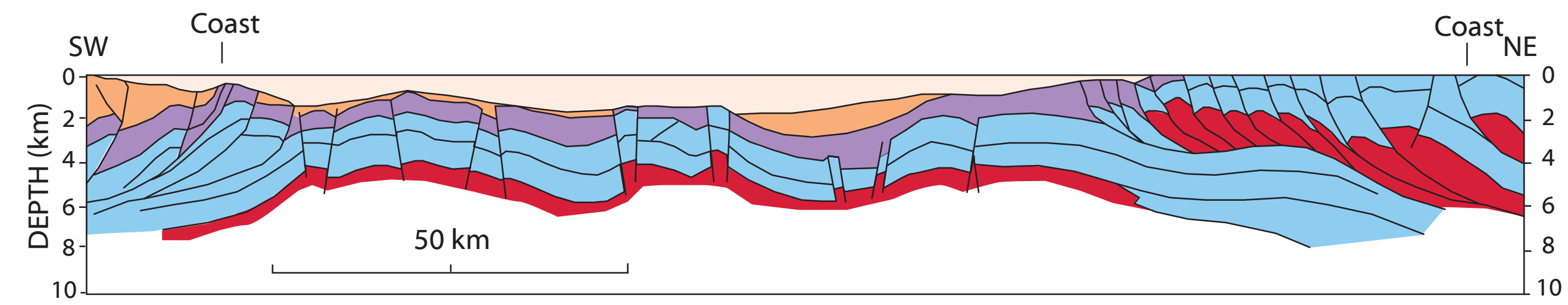


1950s as reflection seismic data were refined. First Italian offshore exploration began in 1960 and first Croatian offshore exploration in 1967. From the early 1950s until the mid 1990s, AGIP SpA - the Italian state oil company - had exclusive exploration rights in most of the Italian Po Basin Province.

Early onshore exploration was for shallow, Tertiary-reservoired structural anticlines identified with seismic data. Deeper Mesozoic folded and faulted reservoirs were targeted in the 1970s and 1980s, with improved success rates achieved through the use of 3-D seismic techniques.

A total of 2,670 wells are in the Po Basin Province, 97\% in Italy and 3\% in Croatia (Petroconsultants, 1996). Of Italy's wells, $42 \%$ are classified as wildcats (22\% success rate), $41 \%$ as development (78\% success rate) and $17 \%$ as outposts (43\% success rate). Of Croatia's wells (all offshore), 53\% are wildcats (9\% success rate) and $47 \%$ are outposts ( $28 \%$ success rate).

\section{PETROLEUM OCCURRENCE AND CHARACTER}

There are 263 fields in the Po Basin Province (Table 1), with 3.54 BBOE (48\% produced) known, ultimately recoverable reserves (Petroconsultants, 1996) in three total petroleum systems. Ninety-seven percent of those ultimately recoverable reserves are in Italy; $3 \%$ are in Croatia. Onshore, 160 fields are in Italy and none in Croatia. The westernmost Italian onshore area and the Italian Veneto Plain north of the Adriatic Sea contain few fields (Figure 1). Offshore, 100 fields are distributed with 13 in Croatia and 87 in Italy. (Another three Italian fields discussed in the literature are not in the Petroconsultants data base of "fields.") Eighty-eight percent of the total province reserves are gas (nearly 19 TCF), and 50\% of the gas has been produced. Eleven percent of the total province known or discovered reserves are oil (nearly $400 \mathrm{MMBOE}$ ), with $24 \%$ of the oil having been produced. One percent of the total province reserves is condensate, and $83 \%$ of that has been produced. Reservoir rocks range in age from Triassic to Quaternary.

\section{Porto Garibaldi : Plio-Pleistocene (and minor Miocene) Biogenic Gas}

Conditions necessary for the formation and entrapment of gas interpreted to be biogenic include adequate total organic carbon (TOC) content, high sedimentation rates, synsedimentary tectonics, adequate reservoir presence, and a cool thermal regime $\left(<60^{\circ} \mathrm{C}\right)$ where bacteria can still exist and where organic matter does not degrade significantly (Tissot and others, 1990). Biogenic gas accumulations are commonly found in close proximity to their organic source material, with negligible migration required. Such conditions were met in the Po Basin Province during deposition of Neogene (especially Pliocene) and Pleistocene siliciclastic turbidites. The optimal location was the Apennine foredeep, northeast of and parallel with the present Apennine thrust front. Although biogenic gas has been confirmed within the entire Po Basin Province from as far west as Milan (Figures 4 and 5a), the best combination of factors for its entrapment is in the eastern half of the province near the coast and offshore. Because of the cool Cenozoic 


\begin{tabular}{|c|c|c|c|c|}
\hline Fi & С Туре & $\overline{c s s}$ & & Loce \\
\hline $\begin{array}{l}\text { Ada } \\
\text { agnadello } \\
\text { agnese } 1 \\
\text { Aida } \\
\text { Alfonsine }\end{array}$ & $\begin{array}{l}G \\
G \\
G \\
G \\
G \\
G\end{array}$ & $\begin{array}{c}\text { B } \\
\text { B? } \\
\text { B } \\
\text { B } \\
B\end{array}$ & & \begin{tabular}{|l} 
OFF \\
on \\
OFF \\
OFF \\
on
\end{tabular} \\
\hline $\begin{array}{l}\text { Amisia Mare } \\
\text { Andreina 1 }\end{array}$ & $\vec{G}$ & $\begin{array}{l}\mathrm{B} \\
\mathrm{B}\end{array}$ & $\frac{1}{x}$ & \begin{tabular}{|l|l} 
OFF \\
OFF
\end{tabular} \\
\hline $\begin{array}{l}\text { Anemone } \\
\text { Angela } \\
\text { Angelina }\end{array}$ & $\begin{array}{l}G \\
G \\
G\end{array}$ & $\begin{array}{l}\text { B } \\
\text { B } \\
\text { B }\end{array}$ & $\begin{array}{l}x \\
x\end{array}$ & \begin{tabular}{|l} 
OFF \\
OFF \\
on
\end{tabular} \\
\hline \begin{tabular}{|l|} 
Anna Maria \\
Annabella \\
Annalisa 1 \\
Antares \\
Antros
\end{tabular} & $\begin{array}{l}G \\
G \\
G \\
G\end{array}$ & $\begin{array}{l}B \\
B \\
B \\
B \\
B\end{array}$ & $\begin{array}{c}x \\
\text { GasAn } \\
\end{array}$ & \begin{tabular}{|l|l|} 
OFF \\
OFF \\
OFF \\
OFF
\end{tabular} \\
\hline 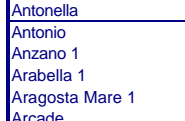 & $\begin{array}{l}G \\
G \\
G \\
G \\
G \\
G\end{array}$ & $\begin{array}{c}\text { B } \\
\text { B } \\
\text { BMio } \\
\text { B } \\
\text { B }\end{array}$ & & \begin{tabular}{|l|} 
OFF \\
OF \\
Of \\
OFF \\
OF
\end{tabular} \\
\hline $\begin{array}{l}\text { Antanna } \\
\text { Armida } \\
\text { Am }\end{array}$ & $\begin{array}{l}G \\
G\end{array}$ & $\begin{array}{l}\mathrm{B} \\
\mathrm{B}\end{array}$ & & \begin{tabular}{|l|l} 
OFF \\
OFF
\end{tabular} \\
\hline $\mid \begin{array}{l}\text { Atitila } \\
\text { Azalea } \\
\text { Azzurara } 1\end{array}$ & $\begin{array}{l}G \\
G \\
G \\
G \\
G\end{array}$ & $\begin{array}{l}\text { B } \\
\text { B } \\
\text { B } \\
\end{array}$ & $x$ & \begin{tabular}{|l} 
OFF \\
OFF \\
OFF \\
\end{tabular} \\
\hline 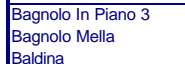 & $\begin{array}{l}\text { Oil } \\
\text { GC } \\
\text { G }\end{array}$ & $\begin{array}{c}\text { TERT } \\
B \\
B\end{array}$ & $\begin{array}{l}x \\
x\end{array}$ & \begin{tabular}{|l} 
on \\
on \\
on
\end{tabular} \\
\hline 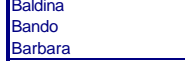 & $\begin{array}{l}G \\
G \\
G\end{array}$ & $\begin{array}{l}B \\
B \\
B\end{array}$ & & \begin{tabular}{|l} 
on \\
on \\
OFF
\end{tabular} \\
\hline 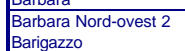 & $G$ & $\begin{array}{l}B \\
B \\
B P 2\end{array}$ & & \begin{tabular}{|l|} 
OFF \\
on \\
\end{tabular} \\
\hline 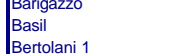 & $\begin{array}{l}G \\
G \\
G\end{array}$ & $\begin{array}{l}\text { B? } \\
B \\
B ?\end{array}$ & & \begin{tabular}{|l} 
On \\
OFF \\
on
\end{tabular} \\
\hline 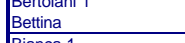 & $\mathrm{G}$ & B & & \begin{tabular}{|l} 
on \\
OFF \\
\end{tabular} \\
\hline 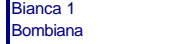 & $\begin{array}{l}G \\
G\end{array}$ & $\begin{array}{c}\text { B? } \\
\text { TERT }\end{array}$ & & \begin{tabular}{|l|l} 
OFF \\
on
\end{tabular} \\
\hline 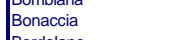 & G & B & & \begin{tabular}{|l|l|l|l} 
OFF \\
\end{tabular} \\
\hline $\begin{array}{l}\begin{array}{l}\text { Bordolano } \\
\text { Bottoni }\end{array} \\
\text { and }\end{array}$ & $\begin{array}{l}\mathrm{GC} \\
\mathrm{G}\end{array}$ & mixed & $x$ & \begin{tabular}{|c} 
on \\
on \\
\end{tabular} \\
\hline $\begin{array}{l}\begin{array}{l}\text { Bova } \\
\text { Brazzuto }\end{array} \\
\end{array}$ & $\begin{array}{l}G \\
G \\
G\end{array}$ & $\begin{array}{l}B \text { B? } \\
B\end{array}$ & & \begin{tabular}{|c|c|} 
on \\
on
\end{tabular} \\
\hline $\begin{array}{l}\text { Brenda } \\
\text { Brugherio }\end{array}$ & ${ }_{G}^{G}$ & $\begin{array}{l}\text { B } \\
\text { B }\end{array}$ & & \begin{tabular}{|l} 
OFF \\
on
\end{tabular} \\
\hline \begin{tabular}{|l} 
Budrio Est \\
Busseto
\end{tabular} & $\frac{G C}{G C D}$ & $\begin{array}{l}\text { mixed } \\
\text { TRTT }\end{array}$ & GasAn & on \\
\hline $\begin{array}{l}\text { Busseto } \\
\text { Ca Bellavista } \\
\text { Ca Boldrini } \\
\text { a Bolvi }\end{array}$ & $\begin{array}{c}\text { GCO } \\
G \\
G\end{array}$ & $\begin{array}{l}\text { TERT } \\
\text { TERT } \\
\text { B? }\end{array}$ & $x$ & \begin{tabular}{|l} 
on \\
on \\
on
\end{tabular} \\
\hline $\begin{array}{l}\text { Ca Dell'Onesta } 2 \\
\text { Caleppio }\end{array}$ & $\begin{array}{l}G \\
G \\
G\end{array}$ & $\begin{array}{l}\mathrm{B} ? \\
\mathrm{~B}\end{array}$ & $x$ & \\
\hline \begin{tabular}{|l} 
Calipso 1 \\
Caluuria
\end{tabular} & $\begin{array}{l}G \\
G\end{array}$ & $\begin{array}{l}\text { B? } \\
\text { B }\end{array}$ & & \begin{tabular}{|l} 
OFF \\
OFF
\end{tabular} \\
\hline $\begin{array}{l}\text { appumia } \\
\text { Carlo }\end{array}$ & $\mathrm{G}$ & B & & \begin{tabular}{|l}
$\mathrm{OFF}$ \\
$\mathrm{OFF}$
\end{tabular} \\
\hline \begin{tabular}{|l} 
Carolal 1 \\
Casalmorano 1
\end{tabular} & $\begin{array}{l}G \\
G \\
G\end{array}$ & $\begin{array}{c}\text { B } \\
B ?\end{array}$ & & \begin{tabular}{|l} 
OFF \\
on \\
\end{tabular} \\
\hline $\begin{array}{l}\begin{array}{l}\text { Castagano } \\
\text { Casteggio }\end{array} \\
\end{array}$ & $\begin{array}{l}G \\
G \\
G\end{array}$ & $\begin{array}{c}\text { TeET } \\
\text { mixed }\end{array}$ & $x$ & \begin{tabular}{|l} 
on \\
on
\end{tabular} \\
\hline 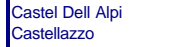 & $\frac{O G C}{G}$ & $\begin{array}{l}\text { TERT } \\
\text { B? }\end{array}$ & & \begin{tabular}{|l|} 
on \\
on
\end{tabular} \\
\hline \begin{tabular}{|l|l|l|l|l} 
Cavalleto \\
Caaviaga
\end{tabular} & $\frac{G}{G C}$ & $\begin{array}{l}\text { BMio } \\
\text { mixiod }\end{array}$ & $x$ & \begin{tabular}{|l} 
on \\
on
\end{tabular} \\
\hline $\begin{array}{l}\text { Cavone } \\
\text { Conte }\end{array}$ & Oil & Tri & $\mathrm{x}$ & \begin{tabular}{|l|l} 
on \\
0
\end{tabular} \\
\hline $\mid \begin{array}{l}\text { Cecilia } 1 \\
\text { Cernusco }\end{array}$ & $\begin{array}{c}G \\
G C\end{array}$ & $\begin{array}{l}\text { B? } \\
\text { Tri }\end{array}$ & $\mathrm{x}$ & \begin{tabular}{|l} 
OFF \\
on
\end{tabular} \\
\hline 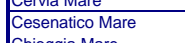 & $\frac{G}{G}$ & $\frac{B}{B}$ & & \\
\hline \begin{tabular}{|l}
$\mid \begin{array}{l}\text { Chioggia Mare } \\
\text { Cignone }\end{array}$ \\
|
\end{tabular} & $\begin{array}{l}G \\
G\end{array}$ & $\begin{array}{l}\text { B } \\
\text { B? }\end{array}$ & & \begin{tabular}{|l|} 
OFF \\
on
\end{tabular} \\
\hline $\begin{array}{l}\text { Clara Est } \\
\text { Clara Nord } 1 \\
\end{array}$ & $\begin{array}{l}G \\
G \\
\end{array}$ & $\begin{array}{l}\text { B? } \\
\text { B? }\end{array}$ & & \begin{tabular}{|l} 
OFF \\
OFF \\
\end{tabular} \\
\hline \begin{tabular}{|l} 
Clarar Ovest \\
Claudia 1
\end{tabular} & ${ }_{G}^{G}$ & $\begin{array}{l}\mathrm{B} ? \\
\mathrm{~B}\end{array}$ & & \begin{tabular}{|l} 
OFF \\
OFF
\end{tabular} \\
\hline $\mid \begin{array}{l}\text { Collecchio } \\
\text { Colombarala } 1\end{array}$ & ${ }_{G}^{G}$ & $\begin{array}{c}B \\
\text { mixed? }\end{array}$ & $\mathrm{x}$ & \begin{tabular}{|l|l|} 
on \\
on
\end{tabular} \\
\hline $\begin{array}{l}\text { Dommachoria } \\
\text { Core } 1 \\
\end{array}$ & $\begin{array}{l}a \\
G \\
G\end{array}$ & $\begin{array}{l}\text { mixed? } \\
\text { B? } \\
\end{array}$ & & \begin{tabular}{|l} 
on \\
OFF \\
\end{tabular} \\
\hline $\begin{array}{l}\text { Congegliano } \\
\text { Consandolo }\end{array}$ & ${ }_{G}^{G}$ & $\begin{array}{l}\text { BMio } \\
\text { B? }\end{array}$ & & \begin{tabular}{|l} 
on \\
on
\end{tabular} \\
\hline \begin{tabular}{|l} 
Conterico 1 \\
Comegliano
\end{tabular} & $\underset{G C}{G}$ & $\begin{array}{l}\text { B? } \\
\text { mixed }\end{array}$ & $x$ & \begin{tabular}{|l|} 
on \\
on
\end{tabular} \\
\hline \begin{tabular}{|l|} 
Correggio \\
Contemagaiore
\end{tabular} & $\frac{G C}{O G C}$ & B & 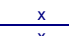 & \begin{tabular}{|l|l} 
on \\
\end{tabular} \\
\hline 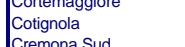 & $\begin{array}{c}\text { OGC } \\
G \\
G C\end{array}$ & $\begin{array}{l}\text { TERT } \\
\text { B } \\
\text { TEPT }\end{array}$ & $\begin{array}{l}x \\
x \\
x\end{array}$ & \begin{tabular}{|l} 
on \\
on
\end{tabular} \\
\hline $\begin{array}{l}\text { Tusanono Mulanono } \\
\text { Dacia }\end{array}$ & $\begin{array}{l}\mathrm{GC} \\
G \\
G\end{array}$ & $\begin{array}{l}\text { TEET } \\
\text { B? } \\
\text { B? }\end{array}$ & & \begin{tabular}{|l} 
on \\
on \\
OFF
\end{tabular} \\
\hline Daria & $G$ & $B$ & & \\
\hline $\mid$\begin{tabular}{|l} 
Debora \\
Delia
\end{tabular} & $\begin{array}{l}G \\
G\end{array}$ & $\begin{array}{l}B \\
B\end{array}$ & & \begin{tabular}{|l} 
OFF \\
OFF
\end{tabular} \\
\hline $\begin{array}{l}\text { Demetra } \\
\text { Docana }\end{array}$ & $G$ & B & & OFF \\
\hline 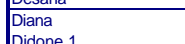 & $G$ & B & & \begin{tabular}{|l|l|} 
OFF \\
OFEF
\end{tabular} \\
\hline $\mid \begin{array}{l}\text { idonen } 1 \\
\text { Donatella }\end{array}$ & $G$ & $\begin{array}{l}B \\
B\end{array}$ & & \begin{tabular}{|l} 
OF- \\
OFF
\end{tabular} \\
\hline \begin{tabular}{|l} 
Donatella Ovest 1 \\
Dorotea
\end{tabular} & $\begin{array}{l}G \\
G \\
G\end{array}$ & $\begin{array}{l}B \\
B\end{array}$ & & \begin{tabular}{|l} 
OFF \\
OFF
\end{tabular} \\
\hline \begin{tabular}{|l|} 
Dosso degli Angeli \\
Elettra 1
\end{tabular} & $G_{G}$ & $\begin{array}{l}B \\
B ? \\
B ?\end{array}$ & $x$ & \begin{tabular}{|l|l} 
on \\
OFF
\end{tabular} \\
\hline Emanuela & $G$ & B & & OFF \\
\hline $\begin{array}{l}\text { arabila } 1 \\
\text { Eano }\end{array}$ & $\begin{array}{l}G \\
G \\
G\end{array}$ & $\begin{array}{l}\mathrm{B} \\
\mathrm{B} ?\end{array}$ & & \begin{tabular}{|l} 
OFF \\
on \\
\end{tabular} \\
\hline $\begin{array}{l}\text { Fiorllal } 1 \\
\text { Fontevivo-Bellena }\end{array}$ & $\begin{array}{l}G \\
O G\end{array}$ & $\begin{array}{c}B \\
\text { TERT } \\
\end{array}$ & $x$ & \\
\hline 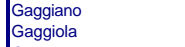 & $\begin{array}{l}\text { Oil } \\
G\end{array}$ & $\underset{\substack{\text { Tri } \\
\text { TERT }}}{ }$ & $x$ & \begin{tabular}{|l|} 
on \\
on
\end{tabular} \\
\hline Gaia & $\frac{G}{G}$ & $\frac{B}{B}$ & 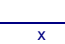 & \\
\hline $\begin{array}{l}\text { Gallare } \\
\text { Gallignano } 2\end{array}$ & $G$ & B? & A & \begin{tabular}{|l|} 
on \\
on
\end{tabular} \\
\hline 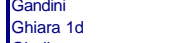 & ${ }_{G}^{G}$ & $\begin{array}{c}B \\
\text { TERT }\end{array}$ & $x$ & \begin{tabular}{|l} 
on \\
on
\end{tabular} \\
\hline Gilivia & $G$ & $\begin{array}{ll}8 ? \\
B ?\end{array}$ & & \begin{tabular}{|l|l|} 
OFF \\
of
\end{tabular} \\
\hline $\begin{array}{l}\text { Greecchia } \\
\text { Grignano Polesine }\end{array}$ & ${ }_{G}^{G}$ & $\begin{array}{l}\text { B? } \\
\text { B? }\end{array}$ & & \begin{tabular}{|l} 
on \\
on
\end{tabular} \\
\hline 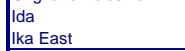 & $G_{G}^{G}$ & $\begin{array}{l}\text { B? } \\
\text { B? }\end{array}$ & & \begin{tabular}{|l} 
OFF \\
OFF
\end{tabular} \\
\hline $\mathrm{J}_{\mathrm{k}} \mathrm{a}$ West & $\mathrm{G}$ & B? & & \\
\hline $\begin{array}{l}\text { lka a best } \\
\text { llaria } 1\end{array}$ & $G_{G}^{G}$ & $\begin{array}{l}\text { B? } \\
B\end{array}$ & & \begin{tabular}{|l} 
OF- \\
OFF
\end{tabular} \\
\hline limola & $G$ & 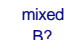 & $x$ & $\begin{array}{l}\text { on } \\
O F=\end{array}$ \\
\hline lima & $G$ & B? & & \begin{tabular}{|l} 
OFF \\
OFF
\end{tabular} \\
\hline IIma 1 & G & B & & Or \\
\hline $\mid \begin{array}{l}\text { Isabella } 1 \\
\text { stra More } 1\end{array}$ & $\begin{array}{l}G \\
G\end{array}$ & $\begin{array}{l}\text { B? } \\
\text { B? }\end{array}$ & & \begin{tabular}{|l} 
OFF \\
OFF \\
\end{tabular} \\
\hline 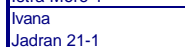 & $G_{G}^{G}$ & $\begin{array}{l}\text { B? } \\
\text { B? }\end{array}$ & & \begin{tabular}{|l|l|} 
OFF \\
OFF
\end{tabular} \\
\hline \begin{tabular}{|l} 
Koralika \\
KKnana
\end{tabular} & ${ }_{G}^{G}$ & $\begin{array}{l}\text { B? } \\
B ?\end{array}$ & & \begin{tabular}{|l} 
OFF \\
OFF
\end{tabular} \\
\hline $\begin{array}{l}\mid \begin{array}{l}\text { Ksenjija } \\
\text { Lambrate }\end{array} \\
\end{array}$ & $\frac{G}{G}$ & $\frac{\mathrm{B} ?}{\mathrm{~B} ?}$ & & on \\
\hline
\end{tabular}

EXPLANATION

HC Type: $\quad \mathrm{G}=$ gas, $C=c$ condensate, $\mathrm{O}=\mathrm{oil}$
HC Source: $\quad ?=$ less certainty, $B=$ biogenic (Plio-Pleistocene), BMio $=$ biogenic (Miocene), $\quad$ mixed $=$ biogenic and thermal, $\quad$ TERT = thermal Tertiary, Tri $=$ thermal Triassic Confidence: $\mathrm{x}=$ published", isotopic confirmation for biogenic gas; GasAn $=$ gas analysis reports $\mathrm{C} 2+<0.2 \%$ (probable biogenic origin). Without published compositional Location: OFF $=$ offshore, on $=$ onshore of criteria by which field accumulations were assigned to particular source rocks. * Anelli and others, 1996; Elliot and others, 1993, laniello and others, 1992; Mattavelli and Maragarucci, 1992; Mattavelli and Novelli, 1988; Mattavelli and Novelli, 1990; Matta-
velli and others, 1983; Mattavelli and others, 1991; Nardon and others, 1991; Pieri, 1992; Pierri and Mattavelli, 1986; Rigo, 1991; Riva and others, 1986: Tissot and others, 1990 


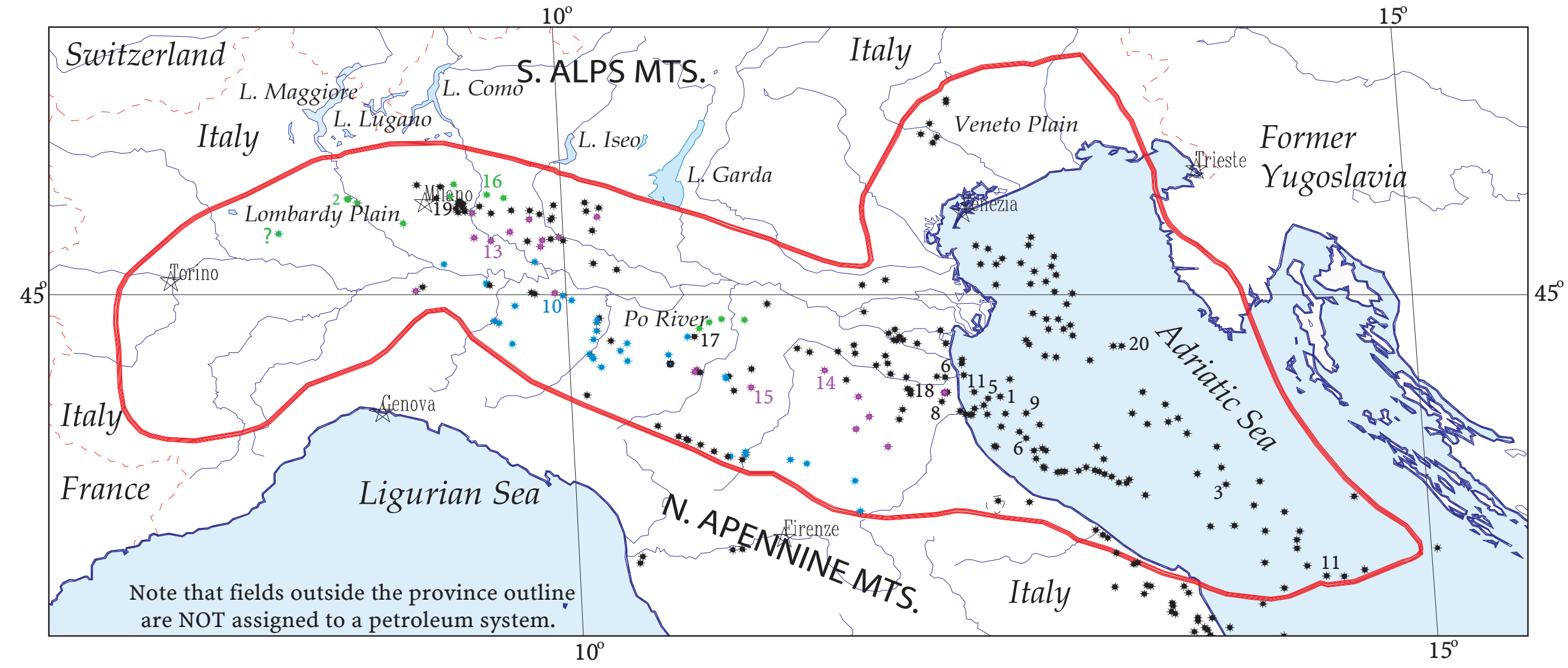

Figure 4. Map of fields color-coded by petroleum system, Po Basin Province \#4060 (Table 1 contains field list).

\section{EXPLANATION}

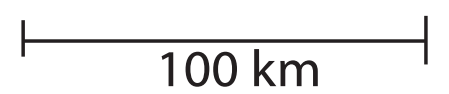

- Rivers

- Shoreline

Po Basin Province boundary Country Boundary
1-20: Top 20 fields by reserves (\#6 and \#11 are 2 fields each with the same reserves)

* Field centerpoints - biogenic gas petroleum system (within province outline only)

* Field centerpoints - mixed biogenic and thermal gas petroleum system

* Field centerpoints - thermal Miocene gas/liquids petroleum system

* Field centerpoints - thermal Triassic liquids/gas petroleum system 


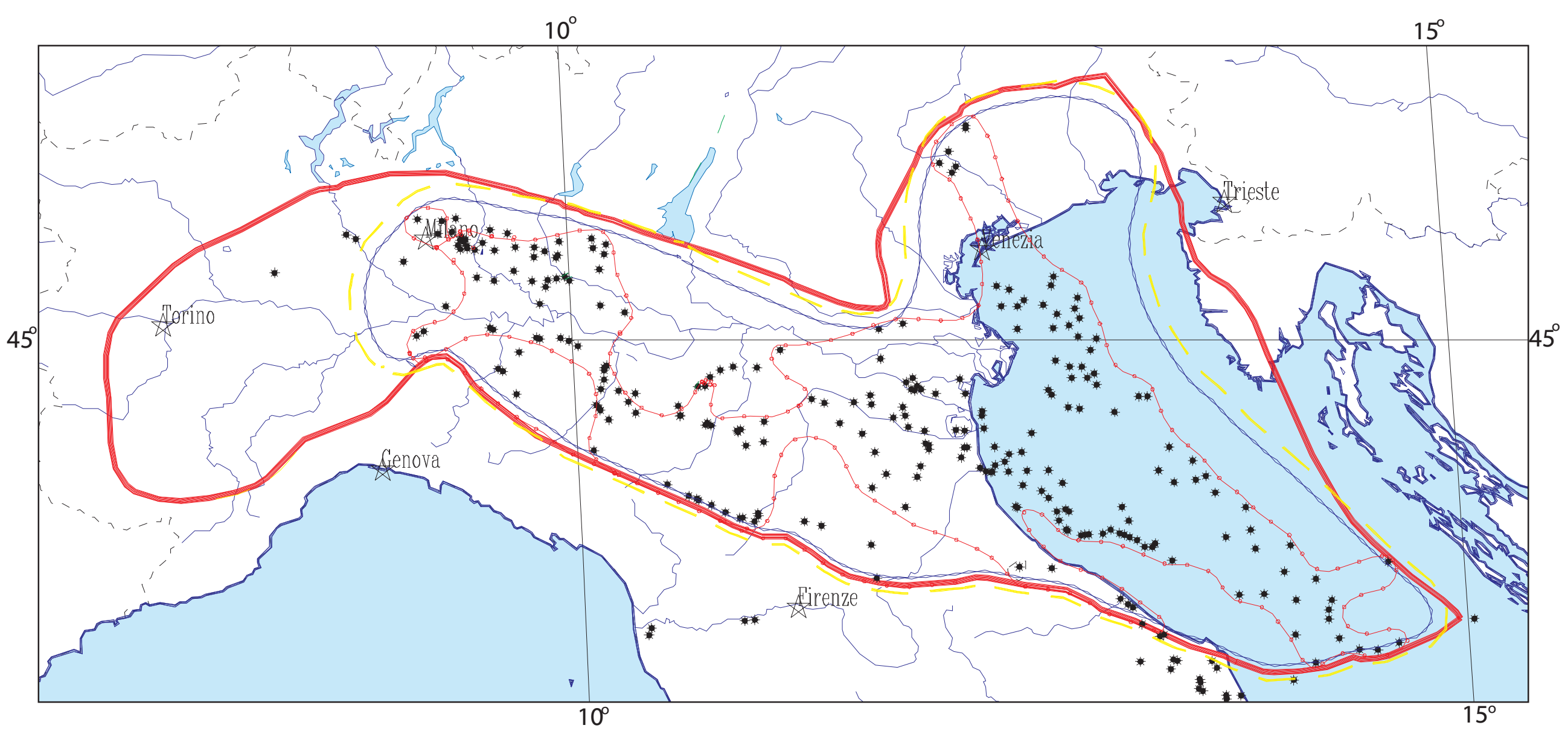

Figure 5a. Porto Garibaldi total petroleum system (Plio-Pleistocene and minor Miocene biogenic gas). This petroleum system would have one biogenic gas assessment unit equal in area to the entire petroleum system. For the quantitative resource assessment, Porto Garibaldi is combined with the volumetrically small Marnoso Arecacea petroleum system (Fig. 5c), still called Porto Garibaldi (406001) with one large assessment unit named Neogene Flysch Gas (40600101). 
thermal regime within the Po Basin Province, confirmed biogenic gas is present at depths

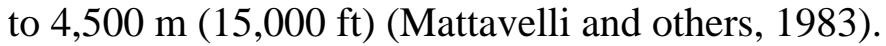

Italian gas of biogenic origin is distinguished from that of thermal origin by the following geochemical criteria incorporating carbon and hydrogen isotopes and $\mathrm{C}_{2}+$ content (Tissot and others, 1990; Mattavelli and others, 1991):

\begin{tabular}{|llll|}
\hline Type of Gas & C isotopes $\left(\delta^{13} \mathrm{C}_{1}\right)$ & H isotopes $(\delta \mathrm{D})$ & $\mathrm{C}_{2+}$ hydrocarbons \\
\hline Biogenic & -75 to $-60 \% \mathrm{Oo}^{-180}$ to $-210 \% \mathrm{oo}$ & $<0.2 \%$ \\
Mixed & -60 to $-50 \%$ oo -180 to $-210 \%$ oo & $0.1-5 \%$ \\
Thermal & -50 to $-30 \%$ oo -150 to $-180 \% \%$ & $0.3-10 \%$
\end{tabular}

Based on the Petroconsultants (1996) data base of known, ultimately recoverable reserves, nearly $85 \%$ (16 TCF) of the total gas (i.e., 75\% of the total hydrocarbons) in the Po Basin Province is gas of biogenic origin (Tables 1 and 2). Another 8-10\% (1.8 TCF) of the province hydrocarbon totals and gas totals is mixed biogenic and thermal gas, with the remaining gas thermally generated. Previous reports attribute a comparable allocation of province gas-in-place reserves to biogenic sources and thermal sources ( $80 \%$ biogenic, $10 \%$ thermogenic and 10\% mixed; Mattavelli and Novelli, 1983).

For this report, all field accumulations have been classified as to a unique or combined hydrocarbon source (Table 1). For the purposes of resource assessment, they are further assigned into just two major petroleum systems. When published designation or compositional information (isotopes or $\mathrm{C}_{1}$ vs. $\mathrm{C}_{2}+$ content) was lacking for a field, the following hierarchy of criteria (data from Petroconsultants, 1996) was used to assign it to a source-rock family:

\begin{tabular}{|c|c|c|c|c|c|}
\hline $\begin{array}{l}\text { Hydrocarbon } \\
\text { Source }\end{array}$ & \multicolumn{2}{|c|}{$\begin{array}{c}\text { Commodity } \\
(\mathrm{G}=\text { gas, } \\
\mathrm{C}=\text { condensate })\end{array}$} & $\begin{array}{c}\text { Field } \\
\text { Location }\end{array}$ & $\begin{array}{r}\text { Reservoi } \\
\text { Depositiol } \\
\text { Environm } \\
\end{array}$ & $\begin{array}{c}\text { Reservoir } \\
\text { Age }\end{array}$ \\
\hline Definite biogenic & \multicolumn{2}{|c|}{ G (plus 2 of following 3 ): } & offshore & deep & Plio/Pleistocene \\
\hline Probable biogenic & \multicolumn{2}{|c|}{ “ (plus 1 of following 3): } & “ & “ & “ \\
\hline Definite mixed & \multicolumn{3}{|c|}{ G or GC (plus all of following 3): onshore } & other than deep & “ \\
\hline Probable mixed & “ & “ & “ & “ & Mio/Plio/Pleistocene \\
\hline Tertiary thermal & “ & “ & “ & “ & Tertiary \\
\hline Triassic thermal & GC or heavier & “ & “ & “ & mostly pre-Tertiary \\
\hline
\end{tabular}


Gas in Miocene reservoirs that is interpreted to be biogenic has been discovered in six fields on the Veneto Plain (Figure 4), but it constitutes $<1 \%$ of total province reserves compared to $>70 \%$ for Plio-Pleistocene biogenic gas (Tables 2 and 3). Five of the six fields produce from Miocene reservoir rocks and one from Pliocene reservoir rocks.

Solely biogenic gas is in 123 fields with Pliocene reservoirs - nearly $50 \%$ of the known recoverable reserves in the province (Table 3) - extending from the Milan area (west) to the Croatian offshore (southeast) (Figures 4 and 5a). The majority of Pliocene reserves (derived from Petroconsultants (1996) data) are in Upper Pliocene rocks, hence the total petroleum system name "Porto Garibaldi" (Figure 2) for both the primary source rock and the primary reservoir rock. Pleistocene biogenic gas reservoirs contain about $21.6 \%$ of the ultimately recoverable province reserves in 74 fields, mostly offshore but also trending intermittently westward to a location near $10^{\circ}$ longitude ( $65 \mathrm{~km}$ east of Milan). A histogram of field sizes for all biogenic gas accumulations is compared with populations for thermogenic and mixed biogenic/thermogenic accumulations in Figure 6. (There were no significant distinctions in field size distribution between Pliocene and Pleistocene reservoirs.) Size data are derived from Petroconsultants (1996).

\section{Mixed Biogenic and Thermal Gas}

Biogenic gas probably mixed with thermal gas occurs in 21 fields (Table 1), generally where tectonism created suitable fault conduits for upward migration of thermal gas (Mattavelli and others, 1983). Fields with potentially mixed accumulations are not areally distinct from those with unmixed accumulations (Figure 4). Nor are size distributions (Figure 6). Potentially mixed accumulations comprise approximately $8.5 \%$ of the total province recoverable reserves (Table 2) and occur primarily in Miocene and younger reservoirs. Statistically, this mixed population is combined with the dominant biogenic gas total petroleum system of the province for the purposes of resource assessment (Figure 5a).

\section{Marnoso Arenacea: Thermal Miocene Gas, Oil and Condensate}

Thermal Miocene hydrocarbons thus far have been discovered solely onshore, mostly in a relatively continuous trend associated with the Apennine foredeep and fold front (Figures 1,4 and 5c) where the thermal gradient is highest in the province. The source rock is the Upper Miocene Marnoso Arenacea Formation (Figure 2). Miocene hydrocarbons are mostly within Tertiary reservoirs in 30 fields (Table 1), hence the total petroleum system name "Marnoso Arenacea" for both the source and reservoir rocks. One field contains a presumed-Tertiary oil of unique geochemical character within a Mesozoic carbonate reservoir (Bagnolo in Piano field; Riva and others, 1986). The Tertiary source rock for that one field is postulated to be older Miocene Gallare marls (Figure 2).

Migration occurred laterally updip from thermally mature synclinal areas and perhaps along faults from thermally mature footwall locations. Volumetrically, thermal Miocene hydrocarbons are mostly gas (Table 2 ), with $\mathrm{C}_{2+}$ content generally higher than in most 
Table 2. Reserve allocations* by hydrocarbon source for the Po Basin Province.

\begin{tabular}{|c|c|c|c|c|c|}
\hline PETROLEUM SYSTEM & $\begin{array}{l}\text { Ultimate Recoverable } \\
\text { Reserves - MMBOE }\end{array}$ & $\begin{array}{l}\% \text { of Province } \\
\text { Total Reserves }\end{array}$ & $\begin{array}{c}\% \text { of Province } \\
\text { Total Gas }\end{array}$ & $\begin{array}{c}\% \text { of Province } \\
\text { Total Oil } \\
\end{array}$ & $\begin{array}{l}\% \text { of URR } \\
\text { Produced }\end{array}$ \\
\hline $\begin{array}{l}\text { Definite Biogenic (Plio-Pleistocene) }(\mathrm{G}) \\
\text { Probable Biogenic (Plio-Pleistocene) (G) }\end{array}$ & $\begin{array}{rr}2388.16 & (14.33 \text { TCF }) \\
253.52 & (1.52 \text { TCF })\end{array}$ & $\begin{array}{c}67.4 \\
7.2\end{array}$ & $\begin{array}{c}75.8^{\star \star \star *} \\
8^{\star \star *}\end{array}$ & $\begin{array}{l}0 \\
0\end{array}$ & $\begin{array}{c}50.57 \\
3.38\end{array}$ \\
\hline Biogenic Miocene (Veneto Plain) (G) & $19.68(.12 \mathrm{TCF})$ & 0.6 & 0.5 & 0 & 50.85 \\
\hline $\begin{array}{l}\text { Definite Mix of Biogenic \& Thermal (G\&C) } \\
\text { Probable Mix of Biogenic \& Thermal (G) }\end{array}$ & 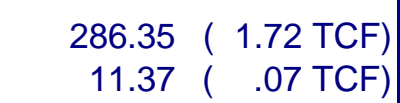 & $\begin{array}{l}8.1 \\
0.3\end{array}$ & $\begin{array}{l}9.1 \\
0.3\end{array}$ & $\begin{array}{l}0 \\
0\end{array}$ & $\begin{array}{l}88.19 \\
20.56\end{array}$ \\
\hline Thermal Tertiary (Miocene) (GC\&O) & (84\% gas) & 4.3 & 4.1 & $6.4^{* *}$ & 63.21 \\
\hline Thermal Triassic (OG\&C) & (16\% gas) & 12.1 & 2.2 & $93.6^{* *}$ & 30.35 \\
\hline
\end{tabular}

* Known ultimately recoverable reserves; data derived from Petroconsultants (1996) and Table 1.

** Stefani and Burchell (1993) report that $70 \%$ of pre-1989 oil in northern Italy is from Triassic source rocks; Mattavelli and Novelli (1990) assign $95 \%$ of Italian oils (all of Italy) to Triassic carbonate source rocks and $5 \%$ to Tertiary shale source rocks.

*** Mattavelli and others (1983) report $80 \%$ of Po Basin gases to be biogenic/diagenetic, $10 \%$ thermal, and $10 \%$ mixed in origin.

$G=$ gas, $O=$ oil, $C=$ condensate (note that condensate comprises just $1 \%$ of total province reserves.) 
Table 3. Ultimate recoverable reserve allocation and average reservoir properties by reservoir age for all Po Basin reserves (data derived from Petroconsultants, 1996).

\begin{tabular}{|c|c|c|c|}
\hline $\begin{array}{c}\text { RESERVOIR } \\
\text { AGE }\end{array}$ & $\begin{array}{c}\% \text { ULTIMATE } \\
\text { REC. RES. }\end{array}$ & $\begin{array}{c}\text { AVERAGE } \\
\text { POROSITY } \\
(\%)\end{array}$ & $\begin{array}{c}\text { AVERAGE } \\
\text { PERMEABILITY } \\
\text { (md) }\end{array}$ \\
\hline $\begin{array}{l}\text { Unallocatable } \\
\text { Unreported }\end{array}$ & $\begin{array}{l}8.69 \\
0.22\end{array}$ & & \\
\hline $\begin{array}{l}\text { CENOZOIC - Quat } \\
\text { Pleistocene (74 fields) }\end{array}$ & 21.63 & 32 & 308 \\
\hline $\begin{array}{l}\text { CENOZOIC - Tert } \\
\text { Pliocene (123 fields) }\end{array}$ & $\begin{array}{l}5^{2} 6.58 \\
49.52\end{array}$ & 31 & 759 \\
\hline Miocene (23 fields) & 1.2 & 29 & 253 \\
\hline Undif. PI-M (10 fields) & 4.29 & 31 & 502 \\
\hline Oligocene (3 fields) & 0.08 & $?$ & $?$ \\
\hline Eocene (3 fields) & 0.06 & $?$ & $?$ \\
\hline Undif. Tert. (18 fields) & 1.43 & $36^{*}$ & $2000^{*}$ \\
\hline MESOZOIC & 12.88 & & \\
\hline Cretaceous (9 fields) & 1.52 & $?$ & $?$ \\
\hline Jurassic (2 fields) & 0.44 & $?$ & $?$ \\
\hline Triassic (5 fields) & 10.24 & 13 & $?$ \\
\hline Undif. (6 fields) & 0.68 & 12 & $?$ \\
\hline
\end{tabular}

* data for 1 reservoir only 


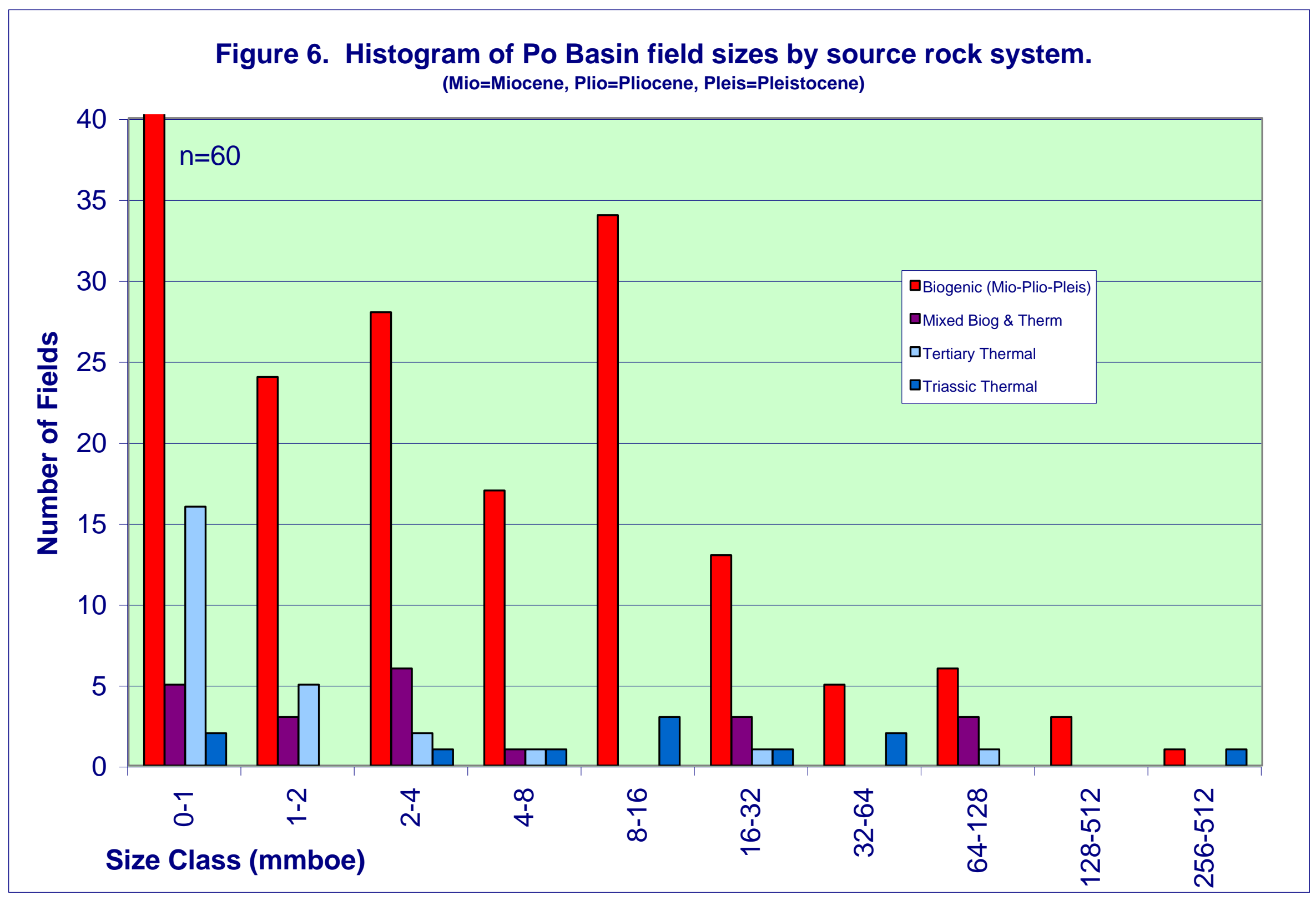




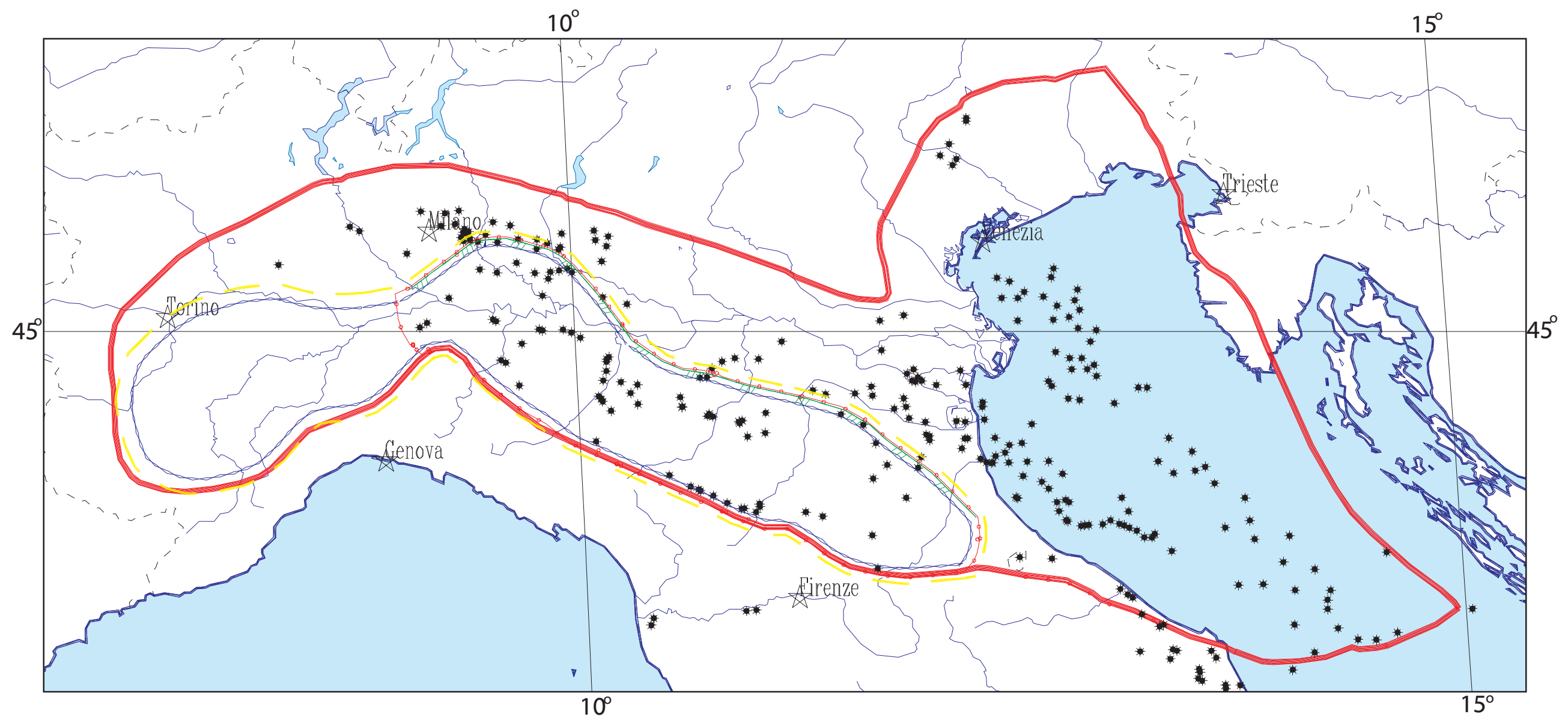

Figure 5c. Marnoso Arenacea total petroleum system (Tertiary thermal gas and condensate). For the purposes of quantitative resource assessment, volumetrically small Marnoso Arenacea is combined with Porto Garibaldi (Fig. 5a), and the resultant total petroleum system is called Porto Garibaldi (406001) with one assessment unit Neogene flysch Gas (40600101).

\footnotetext{
- Rivers

- Shoreline

Po Province boundary

- Country Boundary

* Field centerpoints
}

Projection: Robinson. Central meridian: 0

\section{EXPLANATION}

Assessment regions (collection of polygons)

Minimum Petroleum System regions

Total Petroleum System regions

Pod of active source rocks boundary Tics indicate side of their presence 
biogenic gas (>0.2\%). Thermal Miocene hydrocarbons constitute just $4 \%$ of the total province recoverable reserves (Table 2), and field sizes are smallest in the province (Figure 6).

Miocene-sourced oils from Cortemaggiore field $\left(10^{\text {th }}\right.$-largest province field on Figure 4) are described as napthenic, with API gravities $\left(34^{\circ}-44^{\circ}\right.$ ), sulfur content (maximum $0.14 \%$ ), vanadium content and nickel content similar to thermal oils from Triassic source rocks (Riva and others, 1986; Mattavelli and Novelli, 1990; Pieri, 1992). Miocene oils are distinguished from Triassic oils by unique carbon isotope signatures, high pristane/phytane ratios $(>2)$, the presence of oleanane, and different sterane and hopane distributions. For Miocene oil saturates, $\delta{ }^{13} \mathrm{C}$ ranges from -22.6 to $-23.1 \%$ oo and for aromatics from -22.8 to $-24.4 \%$ oo. The unique Bagnolo-in-Piano (Gallare) oil is heavy (16 ${ }^{\circ} \mathrm{API}$ ) and high in sulfur $(4.9 \%)$. It has $\delta^{13} \mathrm{C}$ values comparable to Miocene Marnoso Arenacea oils but a pristane/phytane ratio intermediate (1.5) between typical Tertiary and Triassic sourced oils. Those parameters correlate well with Gallare source-rock extracts.

\section{Meride / Riva di Solto: Thermal Triassic Oil, Gas and Condensate}

Thermal Triassic-sourced hydrocarbons also have been discovered solely onshore in several distinct areas (Figures 4 and 5b) near or projected from outcrops of organic-rich, Middle to Upper Triassic source-rock facies deposited in anoxic depositional troughs. Triassic hydrocarbons are mostly, but not exclusively, within Mesozoic reservoirs in 12 fields (Table 1). One field (Cernusco) produces known Triassic-sourced hydrocarbons from Pliocene reservoirs. The total petroleum system name reflects the hybrid of 2 major Triassic source rocks - the Middle Triassic (Ladinian) "Meride" Limestone and the Upper Triassic (Rhaetian) "Riva di Solto" Shale (Figure 2). To avoid a cumbersome name length, the largely Mesozoic carbonate reservoirs are excluded from the total petroleum system name.

Lateral updip migration, fault migration, and vertical migration were necessary to charge local structural highs from the areally restricted source rock centers. Volumetrically, Triassic hydrocarbons are mostly oil, and they comprise approximately $12 \%$ of the total province recoverable reserves (Table 2). This thermal Triassic petroleum system has the widest range of field sizes and the flattest distribution (Figure 6).

Oil data have been published for three Triassic-sourced fields that represent the three distinct productive areas - the westernmost Villafortuna-Gaggiano complex (\#2 on Figure 4), Malossa field (\#16 on Figure 4), and Cavone (one of the easternmost Triassic group of four on Figure 4). The oils have distinctions regionally, particularly in terms of API gravity and sulfur content. Villafortuna-Gaggiano oils are overpressured and in a liquid phase at depths of 4600 to 6200 meters (amongst the deepest oil fields in the world). API gravities range from $34^{\circ}-40^{\circ}$, and sulfur content is low (Mattavelli and Novelli, 1990). They are similar to Malossa Triassic oils in carbon isotope values and in isoprenoid distribution, but differ by vanadium/nickel ratio and the presence of dibenzothiophene. 


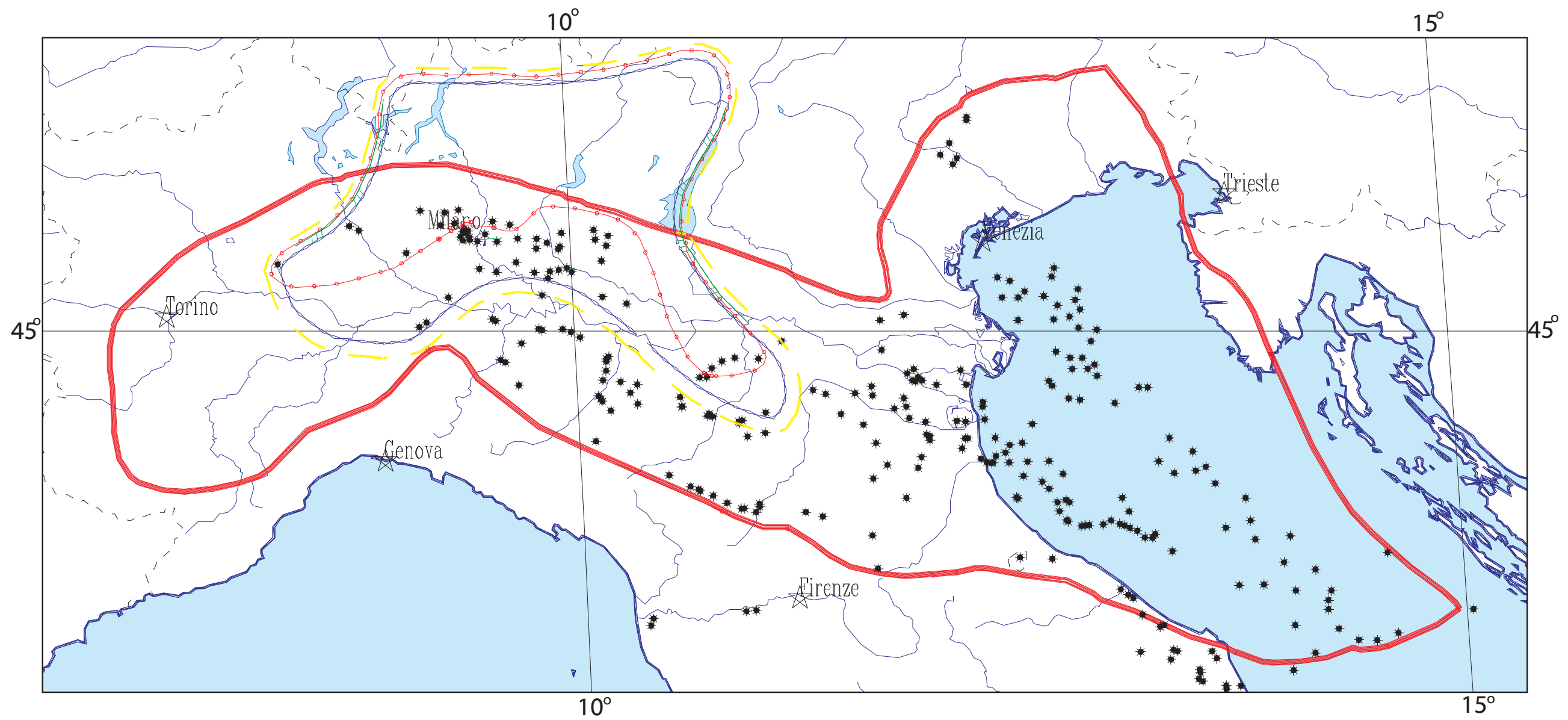

Figure 5b. Meride / Riva di Solto total petroleum system (Triassic thermal oil and gas). This petroleum system has one assessment unit (40600201 ("Thermal Triassic")) equal in area to the entire petroleum system.

\section{EXPLANATION}

- Rivers

$\infty$ Shoreline

Po Province boundary

- Country Boundary

* Field centerpoints

Projection: Robinson. Central meridian: 0
Assessment regions (collection of polygons)

Minimum Petroleum System regions

Total Petroleum System regions

Pod of active source rocks boundary Tics indicate side of their presence 
Malossa oils are also overpressured and have $47^{\circ}-53^{\circ}$ API gravities; low sulfur content $(0$ - $0.6 \%$ ); vanadium, nickel and polar compound presence; a pristane/phytane ratio of 1 1.2 ; and $\delta^{13} \mathrm{C}$ values of -29.2 to $-30.9 \%$ for saturates and -28.5 to $-30.3 \%$ oo for aromatics (Riva and others, 1986; Mattavelli and Novelli, 1990; Stefani and Burchell, 1990). They contain significant quantities of diasteranes and have abundant peaks in $\mathrm{C}_{27}-\mathrm{C}_{35}$ hopanes and $\mathrm{C}_{29}-\mathrm{C}_{30}$ moretanes (Stefani and Burchell, 1990).

Oils from Cavone field have API gravities of $20^{\circ}-22^{\circ}$; high sulfur content (to $4 \%$ ); abundant polar compounds (20-36\%), $\mathrm{C}_{20+}$ content, and biomarkers; negligible diasterane and low Ts/Tm ratios (Riva and others, 1986; Mattavelli and Novelli, 1990).

\section{SOURCE ROCK OCCURRENCE, CHARACTER, AND MATURATION}

\section{Porto Garibaldi: Plio-Pleistocene and minor Miocene Source Rock for Biogenic Gas}

The filling of the Po Basin foredeep occurred in conjunction with the Alpine and Apennine orogenies, resulting in as much as $8 \mathrm{~km}$ of Cenozoic sedimentary fill (Mattavelli and others, 1983). As previously discussed, conditions for the creation, entrapment and preservation of biogenic gas were optimal during deposition of Tertiary and Pleistocene siliciclastic turbidite facies in the Alpine and Apennine foredeep regions, hence the distribution of biogenic gas across nearly the entire Po Basin Province. Thermal gradients over most of the province are presently low, ranging from 19 to $25^{\circ}$ $\mathrm{C} / \mathrm{km}$ (0.7 to $1.4^{\circ} \mathrm{F} / 100 \mathrm{ft}$ ) (Mattavelli and Novelli, 1990), but in the Apennine region they are somewhat higher.

Continued Apennine folding until the Pleistocene Epoch resulted in the Apennine foredeep area being a good location for anticlinal traps, especially the region around the coastline where subsidence was greatest and there was optimal alternation of sandstone and shale for future reservoir and source rock (Figures 1 and 4). In contrast, the Pedealpine region of biogenic gas near Milan (Figures 1 and 4) did not experience such concurrent deformation, and its traps are more stratigraphic. A limited area of Miocene biogenic-gas source rocks from the Alpine foredeep has charged six anticlinal fields in the Veneto Plain of onshore northeastern Italy (Figure 4).

Neogene and Pleistocene bathyal marine shales have total organic carbon (TOC) content ranging from trace amounts to $1.0 \mathrm{wt} \%$ and kerogen composition of $>80 \%$ terrigenous material (Riva and others, 1986; Tissot and others, 1990). These shales are thermally immature (vitrinite reflectance $<0.5 \% \mathrm{R}_{\mathrm{o}}$ ) to depths exceeding $5 \mathrm{~km}$ in the central Po valley. An events chart illustrates the geological recency for all the events and components of this biogenic total petroleum system (Figure 7a). 
Figure 7a. Porto Garibaldi Total Petroleum System Events Chart

Province Name: Po Basin (4060)

TPS Name: Porto Garibaldi

(Mio/Plio/Pleis biogenic gas)

Author(s): S.J. Lindquist

For U.S. Geological Survey resource assessment

Date: April, 1999

—ocal unconformities

purposes, this total petroleum system is combined

with Marnoso Arenacea (Figure 7c).

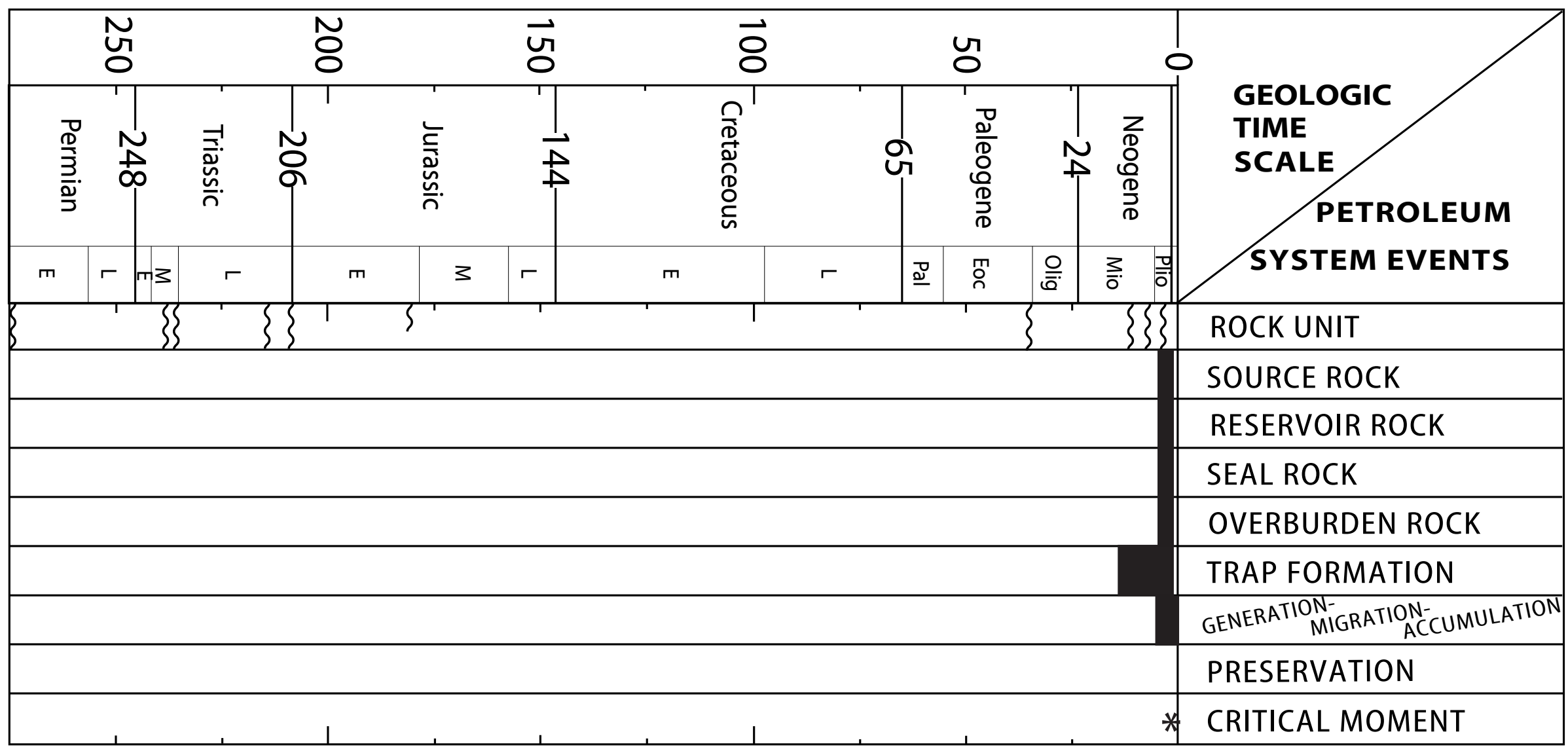




\section{Marnoso Arenacea: Miocene Source Rock for Thermal Gas, Oil and}

Condensate

Miocene bathyal marine shales (and local marls) are thermally immature along the Pedealpine Homocline even to depths of $6 \mathrm{~km}$ (Riva and others, 1986). In the Apennine subsurface fold belt, the oil window is locally as shallow as $4 \mathrm{~km}$, so Miocene thermal hydrocarbon accumulations are largely restricted to the onshore Apennine trend (Figure 4). It is common for Marnoso Arenacea shales to be thermally immature on the tops of anticlines. The formation attains thickness exceeding $3 \mathrm{~km}$ and has average TOC content of $0.68 \mathrm{wt} \%$ and a hydrogen index (HI) of $180 \mathrm{mg} \mathrm{HC} / \mathrm{g}$ TOC (Riva and others, 1986). At 10th-largest Cortemaggiore field, maximum TOCs of $1.5 \mathrm{wt} \%$ and type III kerogens characterize the 2-km thick formation, which generated hydrocarbons in Plio-Pleistocene time (Pieri, 1992). Correlation of oils and source rock extracts (using pristane/phytane ratios, carbon isotopes, and GC/MS data) confirm the Marnoso Arenacea sourcing of numerous fields. An events chart shows a Late Miocene emphasis and importance for events and components of this small Tertiary thermal petroleum system (Figure 7c).

\section{Meride / Riva di Solto: Triassic Source Rock for Thermal Oil, Gas and}

\section{Condensate}

There are published studies of Italian Triassic outcrops across the Southern Alps in a west-to-east trend from Lake Maggiore to the Slovenian border (Figure 1) (Stefani and Burchell, 1993). Triassic shale and carbonate source rocks of variable maturity (immature to highly overmature) crop out in the western half of the province between Lake Lugano (west) and Lake Garda (east). They were deposited in north-south trending, transtensional anoxic basins, several hundred meters deep, interspersed with shallow carbonate platforms. The persistent, Mesozoic platform/graben depositional setting resulted in variable local burial and expulsion histories for the source rock (Figures $7 \mathrm{~b}$ and 8). A Mesozoic overall rise in sea level resulted in the troughs becoming more widespread through time (maximum $1000 \mathrm{sq} \mathrm{km}$; Stefani and Burchell, 1990), such that Rhaetian-age (uppermost Triassic) shales and marls are generally the thickest (>2 km) and most organic rich of the Triassic source rocks. Source rock thickness can vary by an order of magnitude over a $10 \mathrm{~km}$ distance (Mattavelli and Margarucci, 1992).

Thickest and most basinal Upper Triassic source-rock outcrops are located on the west side of Lake Iseo (Figure 4) where they are highly overmature $\left(\mathrm{R}_{\mathrm{o}}=4 \%\right.$, Stefani and Burchell, 1990). Triassic-sourced production closest to Milan (approximately $9.2^{\circ}$ east

longitude, including $16^{\text {th }}$-largest Malossa field) is geochemically tied to Rhaetian euxinic, basinal source rock cropping out directly north of there; whereas the four fields around $11^{\circ}$ east longitude are thought to be charged from organic-rich facies of a postulated small intraplatform depression on the Rhaetian shallow-water carbonate platform cropping out east of and at Lake Garda (Figure 4) (Stefani and Burchell, 1993). If the latter is true, there might be potential for the presence of local Triassic source rocks in the eastern half of the province where only platform carbonates have been identified in outcrop. 
Figure 7c. Marnoso Arenacea Total Petroleum System Events Chart

Province Name: Po Basin (4060)

TPS Name: Marnoso Arenacea

(Tertiary thermal gas, oil, and condensate)

\section{Author(s): S.J. Lindquist}

— Local unconformities
For U.S. Geological Survey resource assessment purposes, this total petroleum system is combined with Porto Garibaldi (Figure 7a).

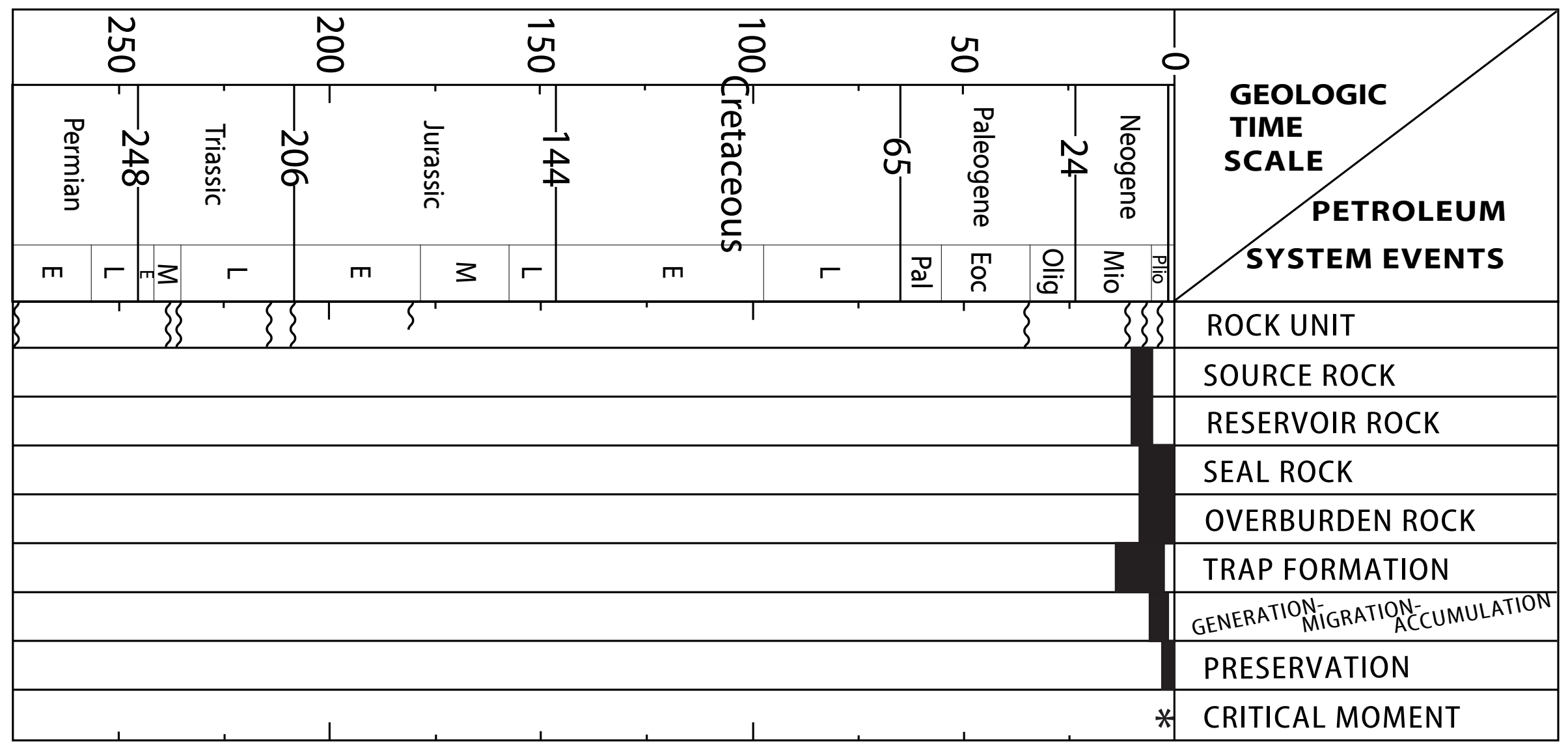


Figure 7b. Meride / Riva di Solto Total Petroleum System Events Chart

Province Name: Po Basin (4060)

Author(s): S.J. Lindquist
TPS Name: Meride / Riva di Solto (406002)

(Triassic thermal oil, gas and condensate)

〜 Local unconformities

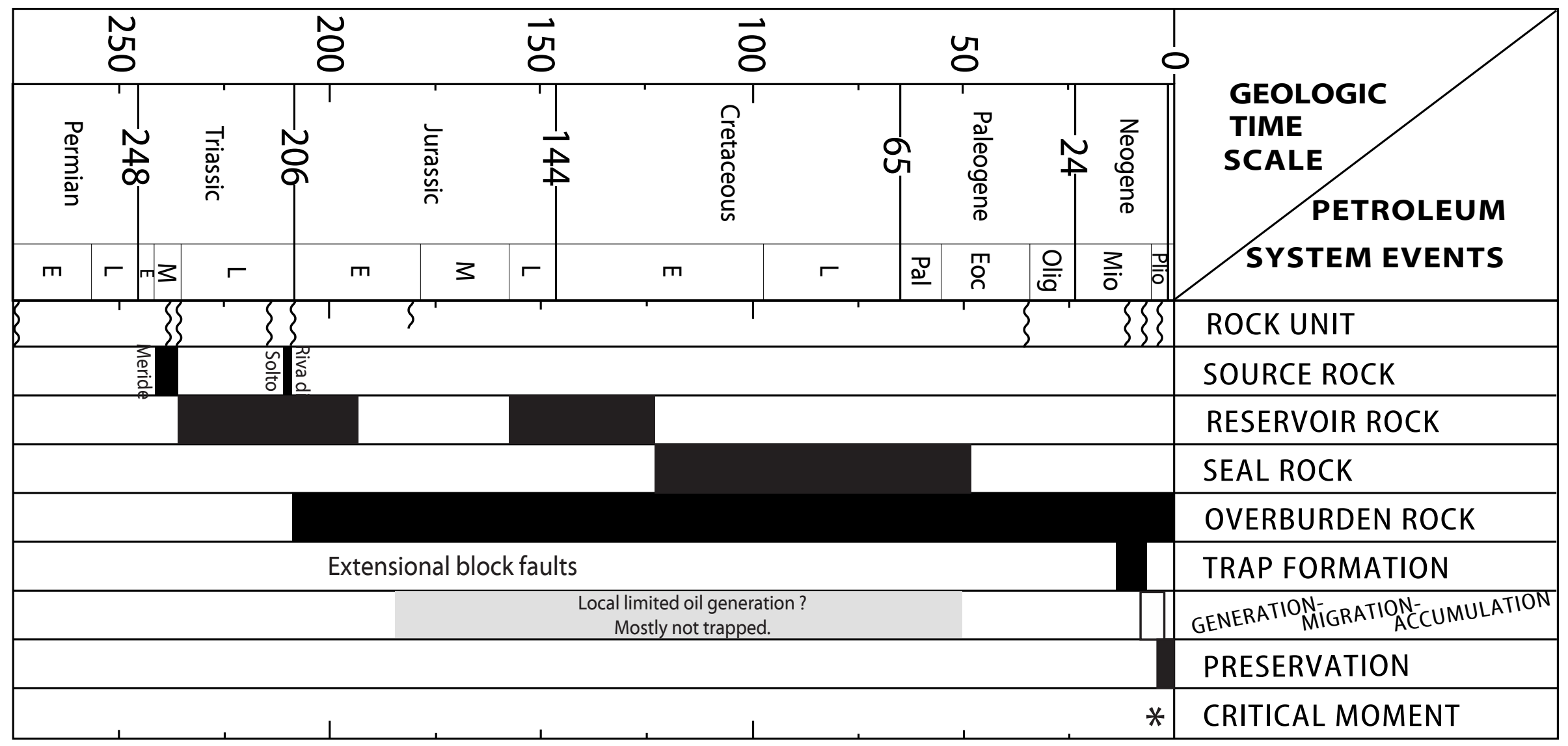


Figure 8. Chart comparing burial histories from two different Triassic producing areas: a shallow burial history for a Middle Triassic source rock (red) and a deeper burial history for an Upper Triassic source rock (green) (after Mattavelli and Margarucci, 1992, and Mattavelli and Novelli, 1990). Also shown is the biogenic gas floor of $4.5 \mathrm{~km}$ (black dotted line) and a schematic burial curve for the basal Miocene foredeep (blue). Apennine foredeep source rocks are thermally mature at shallower depths than Alpine foredeep source rocks and foreland source rocks because of a higher thermal gradient.

GEOLOGIC AGE AND TIME (Ma)

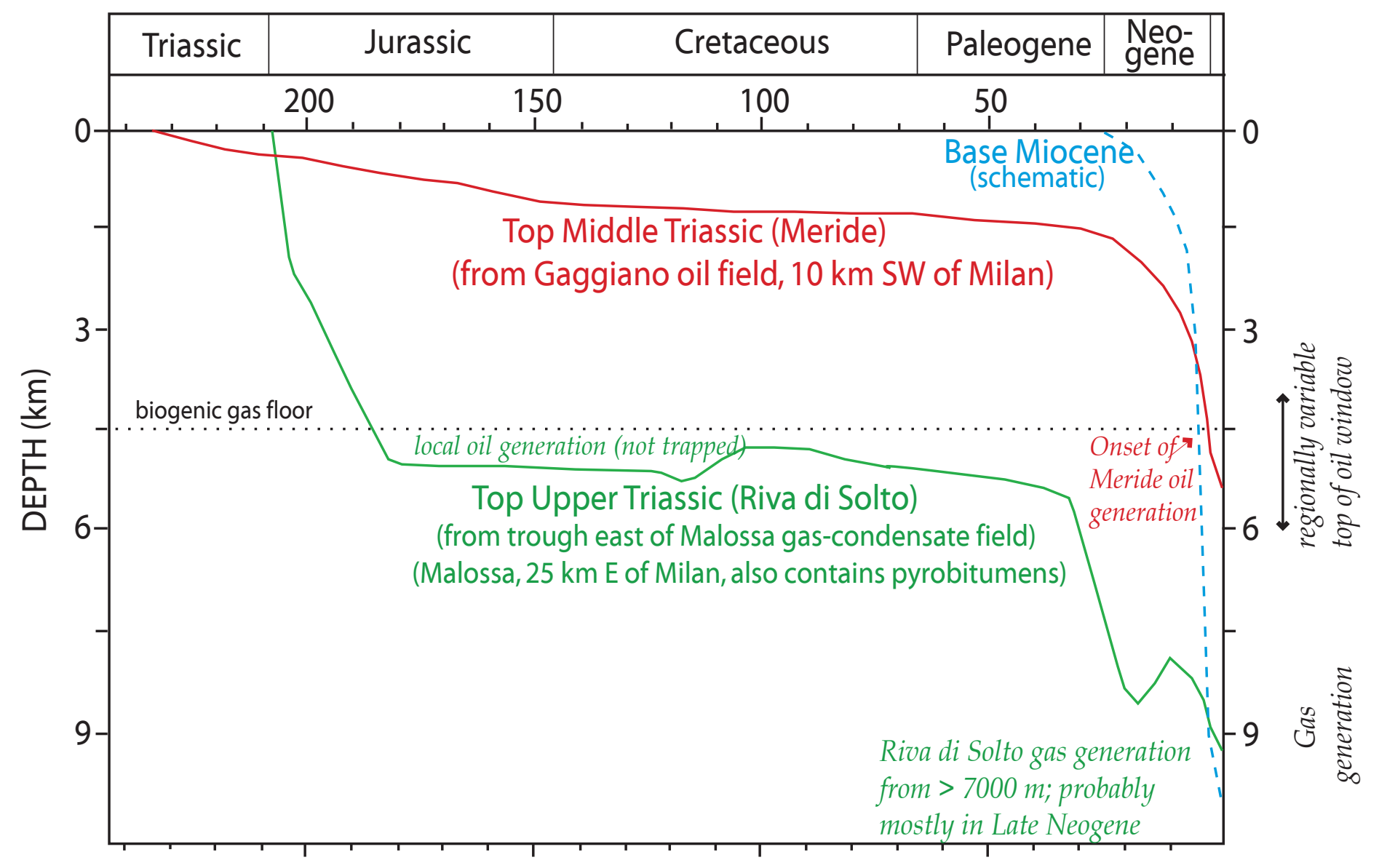


Westernmost Triassic-sourced production from Trecate, Villafortuna $\left(2^{\text {nd }}\right.$ largest province field) and Gaggiano fields is associated with older, Middle Triassic Meride argillaceous carbonate source rocks that crop out in a narrow band between Lake Lugano and Lake Como and that have been penetrated in a Gaggiano well (Riva and others, 1986).

Riva di Solto source-rock extracts are characterized by abundant diasterane content, both marine and continental kerogen types II and III (13-21\% amorphous, 34-59\% herbaceous, $28-45 \%$ woody), and a pristane/phytane ratio near 1 (Stefani and Burchell, 1990, 1993). Land-derived kerogen is probably over-represented in outcrop samples because of high levels of thermal maturity in the most basinal facies. TOC ranges from 0.5 to $5 \mathrm{wt} \%$. Pyrolysis $S_{2}$ values from several samples range from 1-3 kg/ton, with a carbon preference index (CPI) of 1. Riva di Solto and Meride source rocks can be distinguished from each other by carbon isotopes and biomarkers. Riva and others (1986) compare the Triassic source rocks as follows: Meride TOCs average $0.8 \mathrm{wt} \%$, with a sulfur content of $4.5 \%$ and $\mathrm{HI}$ of $513 \mathrm{mg} \mathrm{HC} / \mathrm{g}$ rock; Riva di Solto TOCs average $1.3 \mathrm{wt} \%$, with a sulfur content of $3.1 \%$ and $\mathrm{HI}$ of $251 \mathrm{mg} \mathrm{HC/g}$ rock.

An events chart shows the emphasis and importance for components and events ranging from Jurassic through Neogene age for this Triassic thermal petroleum system (Figure $7 b)$.

\section{OVERBURDEN ROCK AND BURIAL HISTORY}

The total sedimentary column in the Po Basin Province ranges from a minimum of 4-5 $\mathrm{km}$ at its northern Alpine border to a maximum of approximately $12 \mathrm{~km}$ along the Apennine thrust front (Mattavelli and others, 1983). Mesozoic rocks are dominantly carbonates with shales. Oligocene to Recent sandstones and shales can exceed $9 \mathrm{~km}$ in thickness.

Figure 8 illustrates variability in Triassic expulsion history as related to differences in burial history, reflecting the presence of Mesozoic grabens (deep burial histories) and platforms (shallow burial histories). Graben source rocks would likely be more oil prone than source rocks from the platforms and platform margins. Mesozoic thermal gradients during rifting were probably higher than Cenozoic thermal gradients during continental collision. Much of the Triassic oil potentially generated from deepest grabens as early as Early to Middle Jurassic time either was not trapped or was subsequently lost from extensional fault-block traps during the later Alpine and Apennine orogenies. A later major episode of hydrocarbon expulsion under cooler thermal conditions occurred from Miocene and shallow-burial Triassic source rocks during the Alpine and Apennine orogenies. The highest present Cenozoic thermal gradients are associated with the Apennine mountain front. Regionally within the Po Basin Province, the top of the oil window (approximately $0.6 \% \mathrm{R}_{\mathrm{o}}$ ) ranges from about $4 \mathrm{~km}$ to $6 \mathrm{~km}$ in depth today. 


\section{TRAP STYLE}

Mesozoic extensional block faults throughout the Po Basin Province might have served as early traps for Mesozoic-generated Triassic oils from paleograbens. Late Tertiary reactivation of those faults, during the Alpine and Apennine orogenies, locally compromised those pre-existing traps and influenced the trends of later deformation. Tertiary compression ceased in the western Alps before Pliocene time, but continued in the eastern Alps and northern Apennines into the Quaternary Period. Less modification of Mesozoic extensional block faults occurred in foreland regions away from the thrust fronts. Tertiary siliciclastics are locally detached from underlying Mesozoic carbonates.

Most Mesozoic fields (Meride / Riva di Solto thermal total petroleum system) are in faulted, Mesozoic-carbonate paleohighs that have been modified into basement-involved, thrusted anticlines - south-verging in the southern Alps and north-verging in the northern Apennines. Mesozoic reservoirs of this total petroleum system contain $12+\%$ of the ultimately recoverable province reserves (Tables 2 and 3). Although some traps existed in Mesozoic time, the Neogene Alpine and Apennine orogenies were the critical events for trap formation/modification and for generation and migration (Figure 7b).

Tertiary (largely Miocene) reservoired fields of the thermal Marnoso Arenacea total petroleum system are in faulted, thrusted anticlines characterized with depositional lenses, onlaps and truncations. Faults provide necessary conduits for charging the reservoirs with thermal hydrocarbons generated off-structure. This Tertiary thermal hydrocarbon system contains just $4 \%$ of the province's ultimately recoverable reserves (Table 2). Critical elements and processes for this petroleum system are Neogene in age (Figure 7c).

Tertiary and Quaternary reservoirs of the Porto Garibaldi biogenic-gas total petroleum system are in thrusted anticlines, simple drape structures and stratigraphic traps. Purely stratigraphic traps are underexplored. The presence of major faults increases the possibility that biogenic gas will be mixed with thermal hydrocarbons from below. More than $75 \%$ of the province ultimately recoverable reserves are in traps incorporating biogenic gas (Table 2). Critical elements and processes for this petroleum system are Pliocene and younger in age (Figure $7 \mathrm{a}$ ).

\section{DISCOVERY HISTORY}

The first Italian onshore field was discovered in 1890 - followed by one in 1907 and two in the 1920s - in the Emilia fold trend of the northern Apennine Mountains. These early discoveries were shallow Tertiary anticlines $<1 \mathrm{~km}$ deep and sourced with thermal Miocene gas. Around 1940, the first field sourced from biogenic gas was discovered onshore near the Po River delta. Cernusco, the first Triassic-sourced field, was 
discovered in 1958. Regular annual Italian onshore discoveries began in 1951. The first Italian offshore field discovery was in 1960. The largest province field (Porto Garibaldi Agostino) was discovered offshore in 1968. The first two Croatian (offshore) fields were discovered in 1977, including the largest Croatian field to date, Ivana, which ranks $20^{\text {th }}$ in the province.

\section{RESERVOIR ROCKS}

Mesozoic reservoir rocks in the Po Basin Province are predominantly north-south trending platform carbonates of various genesis and texture. Most Mesozoic reserves are in Triassic rocks (Table 3). Triassic reservoirs are underexplored in the eastern half of the province. Intertidal dolomites at $16^{\text {th }}$ largest Malossa field are characterized with porosity ranging from 1-13\% and permeability averaging in the 10s of millidarcies from moldic, intercrystalline, fracture and vug pore systems (Mattavelli and Margarucci, 1992). Malossa average net reservoir thickness is $300 \mathrm{~m}$ (maximum $580 \mathrm{~m}$ ). At Cavone field multiple reservoirs produce, with net thicknesses ranging from approximately $10 \mathrm{~m}$ to > $100 \mathrm{~m}$ (Nardon and others, 1991). Shallow platform carbonates at Cavone field average $5 \%$ porosity and $60 \mathrm{md}$ matrix permeability. Oolitic limestones have $10 \%$ intergranular porosity and 100 md matrix permeability. Deep-marine carbonate breccias with $9 \%$ average porosity and $10 \mathrm{md}$ average matrix permeability also produce. Fractures can increase permeability by as much as an order of magnitude in these carbonates. Mesozoic carbonate reservoirs in the Po Basin Province contain $12+\%$ of the total ultimate recoverable reserves (Table 3 ).

Tertiary and Quaternary siliciclastic reservoir rocks are predominantly turbidite deposits of the Alpine and Apennine foredeeps and forelands, which cover most of the province. Reservoir rocks range from silty, fine-grained sandstones to local conglomerates that were commonly deposited along the long axes of syndepositional synclines. Onlaps and truncations are common. At onshore Cortemaggiore field (thermal Marnoso Arenacea total petroleum system and $10^{\text {th }}$ largest field in the province), the stacked Miocene reservoirs average $25-30 \%$ porosity and $80-180$ md permeability, with individual producing zones ranging from 1.5 to $31 \mathrm{~m}$ in thickness (Pieri, 1992). The offshore Barbara field (biogenic Porto Garibaldi total petroleum system and $3^{\text {rd }}$ largest field in the province) produces from southeast trending stacks of Quaternary sandstones of centimeter to meter thicknesses (Ianniello and others, 1992). Best porosities are around 30\%, and permeabilities range from 5 to $1000 \mathrm{md}$. Water-free production occurs from sandstones with $35-75 \%$ water saturations and net-to-gross ratios ranging from $27 \%$ in the silty sands to $95 \%$ in the coarser sands. Cenozoic siliciclastic reservoirs in the Po Basin Province contain $>78 \%$ of the total ultimate recoverable reserves (Table 3 ).

\section{SEAL ROCK}

Seals for Mesozoic carbonate reservoirs are at stratigraphic or fault contacts with the uppermost carbonates in the stratigraphic section - Cretaceous-aged, impervious pelagic marls and argillaceous limestones known as Scaglia and Marne di Bruntino (Figure 2). 
Cenozoic siliciclastic reservoirs are sealed by contiguous deep-marine shales and impermeable sandstones, generally meters to tens of meters thick. Some biogenic gas accumulations have shale seals $<1 \mathrm{~m}$ thick and are presumed to be leaking even as they are continually replenished.

\section{ASSESSMENT UNITS}

\section{Porto Garibaldi (Biogenic*) Total Petroleum System \#406001}

One established, biogenic gas assessment unit is appropriate for Porto Garibaldi (Figure 5a). (Examinations of existing field size distributions by reservoir age and by geographic and tectonic location did not distinguish unique populations for the purposes of this resource assessment.) This biogenic-gas petroleum system has an area of approximately $89,300 \mathrm{sq} \mathrm{km}$. Sixty-one percent of the "biogenic gas" assessment unit is onshore; $39 \%$ is offshore. Thirty percent of the offshore is in Croatian waters, and all water depths are $<100 \mathrm{~m}$. Dry methane gas $\left(\mathrm{C}_{2+}<0.2 \%\right)$ is the expected resource, and minimum field size is expected to be 1 mmboe onshore and perhaps 2 mmboe offshore. Further infrastructure development is needed offshore. Purely stratigraphic traps are underexplored within this petroleum system. *For the practical purposes of quantitative resource assessment, Porto Garibaldi is combined with volumetrically small Marnoso Arenacea (discussed below) - but still called Porto Garibaldi (\#406001) - with one established assessment unit named Neogene Flysch Gas (\#40600101) in the U.S. Geological Survey database. In combination, Porto Garibaldi and Marnoso Arenacea contain approximately $18.5 \mathrm{TCF}$ known, ultimately recoverable reserves $(87.9 \%$ of the total province reserves, $97.8 \%$ of the total province gas, and $6.4 \%$ of the total province oil).

\section{Meride / Riva di Solto (Thermal Triassic) Total Petroleum System \#406002}

One established assessment unit (“Thermal Triassic” \#40600201) is utilized for Meride / Riva di Solto (Figure 5b), even though several distinct productive areas are delineated and several different pods of source rock of variable Triassic age are known to exist. Meride / Riva di Solto has an area of approximately 31,500 sq km. The petroleum system and assessment unit are totally onshore in Italy, but the reader should be aware that a largely untested Triassic graben might exist offshore from the area of the Italian Po River delta southeastward (parallel with, but southwest of, the Adriatic axis) (Grandic and others, 1997). The expected resource for Meride / Riva di Solto is oil (possibly overpressured) with associated gas and condensate. Because of potentially great depths, this petroleum system is also underexplored. It is expected that the minimum field size will be 2 mmboe. Meride / Riva di Solto's outline includes the westernmost field (Desana) in the province, of which little is published other than its 1954 discovery date, its abandoned status, and the fact that it has few reserves. Desana could possibly belong to the Marnoso Arenacea total petroleum system instead, so Marnoso Arenacea's outline is in close proximity to Desana field (Figure 5c). 


\section{Marnoso Arenacea (Thermal Tertiary) Total Petroleum System}

For the practical purposes of quantitative resource assessment, volumetrically small Marnoso Arenacea is combined with Porto Garibaldi (discussed above) - and called Porto Garibaldi (\#406001) - with just one established assessment unit named Neogene Flysch Gas (\#40600101) in the U.S. Geological Survey database. Marnoso Arenacea has an area of approximately 35,400 sq km and is entirely onshore in Italy (Figure 5c). The eastern two-thirds, east of Milan, include the Emilia, Ferrara and Romagna fold trends of the northern Apennines Mountains and contain all known production (Figure 1). These thrusted fold trends contain cores of Tertiary or Mesozoic rocks. The western onethird, west of Milan, includes the Monferrato Arc and S. Piedmont Basin that have little published information. These western folds contain allochthonous Liguride oceanic nappe in their cores (Pieri and Flores, 1996). The western area has fewer than two dozen exploratory wells and no shows reported in the Petroconsultants data base.

\section{SELECTED REFERENCES}

Anelli, L., Mattavelli, L., and Pieri, M., 1996, Structural-stratigraphic evolution of Italy and its petroleum systems, in Ziegler, P.A., and Horvath, F., eds., Peri-Tethys Memoir 2: Structure and Prospects of Alpine Basins and Forelands: Editions du Museum national d'Histoire naturelle, v. 170, p. 455-483.

Dercourt, J., Ricou, L.E., and Vrielynck, B., editors, 1993, Atlas Tethys palaeoenvironmental maps and explanatory notes: Paris, Gauthier-Villars, 307 p., 14 maps, 1 plate.

Elliot, T., Ballentine, C.J., O’Nions, R.K., and Ricchiuto, T., 1993, Carbon, helium, neon and argon isotopes in a Po basin (northern Italy) natural gas field: Chemical Geology, v. 106, p. 429-440.

Environmental Systems Research Institute Inc., 1992, ArcWorld 1:3M digital database: Environmental Systems Research Institute, Inc. (ESRI), available from ESRI, Redlands, CA, scale: 1:3,000,000.

Grandic, S., Boromisa-Balas, E., and Sustercic, M., 1997, Exploration concept and characteristic of the Dinarides stratigraphic and structural model in the Croatian offshore area (Part I): NAFTA Journal, v. 48, no. 4, p. 117-128.

Grandic, S., Boromisa-Balas, E., and Sustercic, M., 1997, Exploration concept and characteristics of the stratigraphic and structural models of the Dinarides in the Croatian offshore area, part II: hydrocarbon consideration: NAFTA Journal, v. 48, no. 89, p. 249-266. 
Haan, E.A., and Arnott, R.J., 1991, The late Tertiary evolution of the Alpine system of the Mediterranean area, in Spencer, A.M., ed., Generation, Accumulation and Production of Europe's Hydrocarbons, Special Publication of the European Association of Petroleum Geoscientists No. 1: Oxford, Oxford University Press, p. 341-354.

Ianniello, A., Bolelli, W., and Di Scala, L., 1992, Barbara Field, Adriatic Sea, offshore Italy, a giant gas field masked by seismic velocity anomaly - a subtle trap, in Halbouty, M.T., ed., Giant Oil and Gas Fields of the Decade 1978-1988: Tulsa, OK, Amercian Association of Petroleum Geologists Memoir 54, p. 265-276.

Klett, T.R., Ahlbrandt, T.A., Schmoker, J.W., and Dolton, G.L., 1997, Ranking of the world's oil and gas provinces by known petroleum volumes: U.S. Geological Survey OpenFile Report 97-463, one CD-ROM.

Magoon, L.B., and Dow, W.G., 1994, The petroleum system, in Magoon, L.B., and Dow, W.G., eds., The Petroleum System - from Source to Trap: American Association of Petroleum Geologists Memoir 60, p. 3-23.

Mattavelli, L., and Margarucci, V., 1992, Malossa Field - Italy, Po Basin, in Foster, N.H., and Beaumont, E.A., Treatise of Petroleum Geology, Atlas of Oil and Gas Fields, Structural Traps VII: Tulsa, OK, American Association of Petroleum Geologists, p. 119-137.

Mattavelli, L., and Novelli, L., 1988, Geochemistry and habitat of natural gases in Italy: Organic Geochemistry (Advances in Organic Geochemistry 1987), v. 13, nos. 1-3, p. 1-13.

Mattavelli, L., and Novelli, L., 1990, Geochemistry and habitat of the oils in Italy: American Association of Petroleum Geologists Bulletin, v. 74, no. 10, p. 1623-1639.

Mattavelli, L., Novelli, L., and Anelli, L., 1991, Occurrence of hydrocarbons in the Adriatic basin in Spencer, A.M., ed., Generation, Accumulation and Production of Europe's Hydrocarbons, Special Publication of the European Association of Petroleum Geoscientists No. 1: Oxford, Oxford University Press, p. 369-380.

Mattavelli, L., Ricchiuto, T., Grignani, D., and Schoell, M., 1983, Geochemistry and habitat of natural gases in Po basin, northern Italy: American Association of Petroleum Geologists Bulletin, v. 67, no. 12, p. 2239-2254.

Nardon, S., Marzorati, D., Bernasconi, A., Cornini, S., Gonfalini, M., Mosconi, S., Romano, A., and Terdich, P., 1991, Fractured carbonate reservoir characterization and modelling: a multidisciplinary case study from the Cavone oil field, Italy: First Break, v. 9, no. 12 , p. 553-565. 
Petroconsultants, 1996, Petroleum Exploration and Production Database: Petroconsultants, Inc., P.O. Box 740619, 6600 Sands Point Drive, Houston, TX 77274-0619, USA or Petroconsultants, Inc., P.O. Box 152, 24 Chemin de la Mairie, 1258 Perly, Geneva, Switzerland.

Pieri, M., 1992, Cortemaggiore Field - Italy, Po Plain, Northern Apennines, in Foster, N.H., and Beaumont, E.A., Treatise of Petroleum Geology, Atlas of Oil and Gas Fields, Structural Traps VII: Tulsa, OK, American Association of Petroleum Geologists, p. 99-118.

Pieri, M., and Flores, G., 1996, Italy to open Po valley to competitive exploration: Oil and Gas Journal, v. 94, no. 11 (March 11), p. 99-102.

Pieri, M., and Mattavelli, L., 1986, Geologic framework of Italian petroleum resources: American Association of Petroleum Geologists Bulletin, v. 70., no. 2, p. 103130.

Rigo, F., 1991, Italy to open 'exclusive' Po basin area in 1992: Oil and Gas Journal, v. 89, no. 21 (May 27), p. 102-106.

Riva, A., Salvatori, T., Cavaliere, R., Ricchiuto, T., and Novelli, L., 1986, Origin of oils in Po Basin, northern Italy: Organic Geochemistry (Advances in Organic Geochemistry 1985), v. 10, p. 391-400.

Stefani, M., and Burchell, M., 1990, Upper Triassic (Rhaetic) argillaceous sequences in northern Italy: depositional dynamics and source potential, in Huc, A.Y., ed., Deposition of Organic Facies, AAPG Studies in Geology \#30: Tulsa, OK, American Association of Petroleum Geologists, p. 93-106.

Stefani, M.M., and Burchell, M.T., 1993, A review of the upper Triassic source rocks of Italy, in Spencer, A.M., ed., Generation, Accumulation and Production of Europe's Hydrocarbons III, Special Publication of the European Association of Petroleum Geoscientists No. 3: Berlin, Springer-Verlag, p. 169-178.

Tissot, B., Mattavelli, L., and Brosse, E., 1990, Trends in organic geochemistry and petroleum exploration in Italy, in Huc, A.Y., ed., Deposition of Organic Facies, AAPG Studies in Geology \#30: Tulsa, OK, American Association of Petroleum Geologists, p. 161-179.

Zappaterra, E., 1994, Source-rock distribution model of the Periadriatic region: American Association of Petroleum Geologists Bulletin, v. 78, no. 3, p. 333-354. 